UNITU-THEP-13/1994

July 1994

hep-ph/9407304

\title{
On the analytic properties of chiral solitons in the presence of the $\omega$-meson
}

\author{
H. Weigel ${ }^{\dagger}$, U. Zückert, R. Alkofer, and H. Reinhardt \\ Institute for Theoretical Physics \\ Tübingen University \\ Auf der Morgenstelle 14 \\ D-72076 Tübingen, Germany
}

\begin{abstract}
A thorough study is performed of the analytical properties of the fermion determinant for the case that the time components of (axial) vector fields do not vanish. For this purpose the non-Hermitian Euclidean Dirac Hamiltonian is generalized to the whole complex plane. The Laurent series are proven to reduce to Taylor series for the corresponding eigenvalues and -functions as long as field configurations are assumed for which level crossings do not occur. The condition that no level crossings appears determines the radius convergence. However, the need for regularization prohibits the derivation of an analytic energy functional because real and imaginary parts of the eigenvalues are treated differently. Consistency conditions for a Minkowski energy functional are extracted from global gauge invariance and the current field identity for the baryon current. Various treatments of the Nambu-Jona-Lasinio soliton are examined with respect to these conditions.

Motivated by the studies of the Laurent series for the energy functional the Euclidean action is expanded in terms of the $\omega$-field. It is argued that for this expansion the propertime regularization scheme has to be imposed on the operator level rather than on an expression in terms of the one-particle eigenenergies. The latter treatment is plagued by the inexact assumption that the Euclidean Dirac Hamiltonian and its Hermitian conjugate can be diagonalized simultaneously. It is then evident that approaches relying on counting powers of the $\omega$-field in the one-particle eigenenergies are inappropriate.

Using the expansion of the action up to second order and employing a parametrical description of the soliton profiles the repulsive character of the $\omega$ meson is confirmed. In the presence of the $\omega$ meson the soliton mass is enhanced by a few hundred MeV.
\end{abstract}

$\dagger$ Supported by a Habilitanden-scholarship of the Deutsche Forschungsgemeinschaft (DFG). 


\section{Introduction and Motivation}

In the past few years the picture of baryons as being soliton solutions in effective meson theories has proven to be quite successful [1]. As an example we refer to the appealing explanation of the almost vanishing matrix element of the axial singlet current for the proton, i.e. the so-called "spin-puzzle" [2].

The investigation of the baryon structure in soliton models was started off by the consideration of the large $N_{C}$ (number of color degrees of freedom) limit [3] of Quantum Chromo Dynamics (QCD), the theory generally accepted to describe processes being subject to the strong interacting. In this limit QCD was shown to be equivalent to an effective theory of weakly $\left(\mathcal{O}\left(1 / N_{C}\right)\right)$ interacting mesons. Witten furthermore conjectured that baryons emerge as (topological) solitons of this meson theory [4]. At small energies (or large distances) this effective theory should only contain the low-lying meson states, e.g. the pseudoscalar would-be Goldstone boson of chiral symmetry $(\pi, K, \eta)$. A further requirement when modeling $\mathrm{QCD}$ was to maintain its symmetries, especially the chiral symmetry and its spontaneous breaking. These ideas were brought to practice by the rediscovery of the Skyrme model [5] by Adkins, Nappi and Witten [6]. The Skyrme model represents an effective theory of the pseudoscalar pions (and kaons in the three flavor generalization) and admits topological soliton solutions. In the spirit of Witten's conjecture the baryon number current, $B_{\mu}$, was identified with the topological current [7].

Soon after this, vector mesons were added to the scenery in a way consistent with chiral symmetry [8, 9, 10]. This has turned out to be especially pleasing since it brought together the fruitful concept of vector meson dominance (VMD) and the soliton picture of baryons [11]. In particular the isoscalar-vector $\omega$-meson was shown to play a crucial role. It is directly connected to the baryon current via the stationary condition which generically reads

$$
\left(D^{\mu \nu}+m_{\omega}^{2} g^{\mu \nu}\right) \omega_{\nu}=g B^{\mu}
$$

Here $D^{\mu \nu}$ denotes a kinetic operator $\left(e . g . \partial^{\mu} \partial^{\nu}-g^{\mu \nu} \partial_{\rho} \partial^{\rho}\right)$, which depends on the specific form of the model and $g$ is a coupling constant. For the time component, $\mu=0$, eqn (1.1) is rather a constraint than an equation of motion. VMD then implies to identify the isoscalar part of the electromagnetic current as [12]

$$
j_{I=0}^{\mu}=\frac{e m_{\omega}^{2}}{g} \omega^{\mu}
$$

with $e$ being the electric charget. Transforming (1.1) to momentum space it is straightforward to verify that the isoscalar radius of the nucleon is given by [11

$$
r_{I=0}^{2}=r_{B}^{2}+\frac{6}{m_{\omega}^{2}}
$$

where $r_{B}^{2}=\int d^{3} \mathrm{rr}^{2} B_{0}$ denotes the intrinsic baryonic radius. The additional contribution $6 / m_{\omega}^{2}$, which is completely due to VMD, is crucial in order to reproduce the experimental value for $r_{I=0}^{2} \approx(0.86 \mathrm{fm})^{2}$.

More recently efforts have been made to derive the effective meson theory from QCD motivated models of the quark flavor dynamics. In this context the model of Nambu and

*As the integral over $D^{\mu \nu} \omega_{\nu}$ vanishes $j_{I=0}^{\mu}$ is properly normalized. 
Jona-Lasinio (NJL) [13] has acquired special attention [14]. The associated Lagrangian

$$
\begin{aligned}
\mathcal{L}_{\mathrm{NJL}}=\bar{q}\left(i \not \supset-\hat{m}^{0}\right) q & +2 G_{1} \sum_{i=0}^{N_{f}^{2}-1}\left(\left(\bar{q} \frac{\lambda^{i}}{2} q\right)^{2}+\left(\bar{q} \frac{\lambda^{i}}{2} i \gamma_{5} q\right)^{2}\right) \\
& -2 G_{2} \sum_{i=0}^{N_{f}^{2}-1}\left(\left(\bar{q} \frac{\lambda^{i}}{2} \gamma_{\mu} q\right)^{2}+\left(\bar{q} \frac{\lambda^{i}}{2} \gamma_{5} \gamma_{\mu} q\right)^{2}\right)
\end{aligned}
$$

is chirally symmetric. Here $q$ denotes the quark spinors, $\hat{m}^{0}$ the current quark mass matrix and $N_{f}$ the number of flavor degrees of freedom. The latter will be taken to be two from now on. As the coupling constants $G_{i}$ carry dimension (mass) ${ }^{-2}$ the model is not renormalizable. This requires regularization and introduces an additional parameter, the cut-off $\Lambda$. For a sufficiently large coupling $G_{1}$ the model indeed exhibits spontaneous breaking of chiral symmetry [13]. This feature is reflected by a non-vanishing quark condensate $\langle\bar{q} q\rangle$.

The Lagrangian (1.4) can be rewritten in terms of composite meson fields by functional integral techniques [15]. This procedure is referred to as bosonization. In order to make these functional integrals well behaved it is mandatory to impose Feynman boundary conditions or, equivalently, to perform these integrals in Euclidean space. In Euclidean space the time -coordinate is analytically continued to $x_{0}=-i x_{4}=-i \tau$. It also implies to continue the time components of the vector $\left(V_{\mu} \sim \bar{q} \gamma_{\mu} q\right)$ and axial vector $\left(A_{\mu} \sim \bar{q} \gamma_{\mu} \gamma_{5} q\right)$ fields

$$
V_{0} \rightarrow i V_{4}, \quad A_{0} \rightarrow i A_{4}
$$

and consider $\tau, V_{4}$ and $A_{4}$ as Hermitian quantities. In Euclidean space the bosonized action finally is given as the sum of a purely mesonic term

$$
\mathcal{A}_{\mathrm{m}}=\int d^{4} x\left(-\frac{1}{4 G_{1}} \operatorname{tr}\left(\Sigma^{\dagger} \Sigma-\hat{m}^{0}\left(\Sigma+\Sigma^{\dagger}\right)+\left(\hat{m}^{0}\right)^{2}\right)+\frac{1}{4 G_{2}} \operatorname{tr}\left(V_{\mu} V^{\mu}+A_{\mu} A^{\mu}\right)\right)
$$

and a fermion determinant

$$
\mathcal{A}_{\mathrm{F}}=\operatorname{Tr} \log \left(i D_{E}\right)=\operatorname{Tr} \log \left(i \not \partial+Y+\gamma_{5} A-\left(P_{R} \Sigma+P_{L} \Sigma^{\dagger}\right)\right)
$$

i.e. $\mathcal{A}_{\mathrm{NJL}}=\mathcal{A}_{\mathrm{F}}+\mathcal{A}_{\mathrm{m}}$. Here $P_{R, L}=\left(1 \pm \gamma_{5}\right) / 2$ are the projectors on right- and left-handed quark fields, respectively. The complex field $\Sigma$ describes the scalar and pseudoscalar meson fields, $S_{i j}=S^{a} \tau_{i j}^{a} / 2$ and $P_{i j}=P^{a} \tau_{i j}^{a} / 2: \Sigma=S+i P$. There is no problem with treating $\mathcal{A}_{\mathrm{m}}$ as it obviously is analytical in the meson fields. This, however, is not the case for $\mathcal{A}_{\mathrm{F}}$. As a matter of fact $\mathcal{A}_{\mathrm{F}}$ is (in Euclidean space) not a real quantity and thus may be decomposed into real $\left(\mathcal{A}_{R}\right)$ and imaginary $\left(\mathcal{A}_{I}\right)$ parts

$$
\mathcal{A}_{R}=\frac{1}{2} \operatorname{Tr} \log \left(\not D_{E} \not D_{E}^{\dagger}\right), \quad \mathcal{A}_{I}=\frac{1}{2} \operatorname{Tr} \log \left(\not D_{E}\left(\not D E_{E}^{\dagger}\right)^{-1}\right)
$$

The above mentioned ultraviolet divergencies are completely contained in $\mathcal{A}_{R}$. the treatment in Euclidean space proves to be pertinent since the operator $D_{E} D_{E}^{\dagger}$ is

\footnotetext{
${ }^{\dagger}$ In order to obtain a finite value for $\mathcal{A}_{I}$ a suitable definition of the functional trace is mandatory. See section 3 for details.
} 
positive definite. Thus the proper-time regularization [16] prescription may be applied to $\mathcal{A}_{R}$ resulting in the replacement

$$
\mathcal{A}_{R} \longrightarrow-\frac{1}{2} \operatorname{Tr} \int_{1 / \Lambda^{2}}^{\infty} \frac{d s}{s} \exp \left(-s D_{E} \not_{E}^{\dagger}\right)
$$

Expanding the fermion determinant in terms of the meson fields and their derivatives leads to an effective meson theory 15. As this object is, by construction, a polynomial in the meson fields the analytic continuation back to Minkowski space is well defined. The resulting meson theory reasonably well describes the physics of the pseudoscalar and vector mesons. Furthermore this theory has many features in common with Skyrme type models which are supplemented by vector mesons.

This effective theory also allows one to express physical quantities like the pion decay constant $f_{\pi}$ in terms of the cut-off $\Lambda$ and the constituent quark mass $m=\langle\Sigma\rangle$. In case the axial meson degrees of freedom are ignored this relation reads in the gradient expansion [15

$$
f_{\pi}^{2}=\frac{N_{C} m^{2}}{4 \pi^{2}} \Gamma\left(0,\left(\frac{m}{\Lambda}\right)^{2}\right) .
$$

In practice the physical value $f_{\pi}=93 \mathrm{MeV}$ will be employed to determine $\Lambda$ for a given constituent quark mass $m$.

Later on it was also demonstrated that the bosonized version of the NJL model contained static soliton solutions which extremize the Minkowski energy functional [17]. For the investigation of static field configurations the introduction of a Dirac Hamiltonian in Euclidean space, $h_{E}$, via

$$
i \beta \not D_{E}=-\partial_{\tau}-h_{E}
$$

is useful because $\left[\partial_{\tau}, h_{E}\right]=0$. The Euclidean energy functional is then expressed in terms of the eigenvalues of $h_{E}$. First calculations were constrained to the pseudoscalar mesons. Subsequently the isovector-vector [18] and axial vector mesons [19 were added. The associated field configurations have vanishing temporal components $V_{4}=0$ and $A_{4}=0$ rendering $h_{E}$ an Hermitian operator. Thus the extraction of a Minkowski energy functional from the one in Euclidean space is straightforward. In this context it should be remarked that especially the inclusion of the axial vector meson provides further support for Skyrme type models because it turns out that then the valence quark orbit joins the Dirac sea and the baryon number is completely carried by the distorted Dirac vacuum |19.

The analytic structure of the energy functional changes drastically when the isoscalar vector $\omega$-meson is included. In this case the soliton configuration involves a non-vanishing temporal component leading to a non-Hermitian operator $h_{E}$. So far three distinct approaches to include the $\omega$-meson into the static energy functional of the NJL model have been discussed in the literature [20]- [24]. It is the main purpose of the present paper to illuminate the role of the $\omega$-meson in the NJL soliton model and to study the behavior of the corresponding fermion determinant under transformations which mediate between Euclidean and Minkowski space. The above mentioned importance of the $\omega$-meson for the description of static baryon properties requires a clarification of the situation.

\footnotetext{
${ }_{\ddagger}^{\ddagger}$ The difference $m-m_{0}=-2 G_{1}\langle\bar{q} q\rangle$ reflects the spontaneous breaking of chiral symmetry.
} 
The remainder of this paper is organized as follows. In section 2 we investigate the analytical properties of the eigenvalues and -vectors of $h_{E}$. In section 3 we briefly review the derivation of the Euclidean energy functional and show that the analytic continuation which should transform this object to Minkowski space does not exist. In section 4 requirements on the Minkowski energy functional from a phenomenological point of view are derived. Furthermore several definitions for the Minkowski energy functional are discussed. Numerical simulations for the eigenvalues of $h_{E}$ show that treating the $\omega$-meson up to quadratic order represents a reasonable approximation. Using this result, it is argued that the same approximation for the Euclidean energy functional is pertinent. Then a Minkowski energy functional can actually be derived. The study of this approximation is presented in section 5. In that section we also demonstrate that counting powers of the $\omega$-field in the eigenvalues of $h_{E}$ leads to incorrect results for the module of the fermion determinant. As a matter fact, these misinterpretations are related to the appearance of $\not D_{E}^{\dagger}$ in the regularized version of the fermion determinant. Concluding remarks may be found in section 6 . Technical details on the perturbative expansion used in section 5 are discussed in an appendix.

\section{Analytic Properties of the Dirac Hamiltonian}

In the introductory section we have argued that it is mandatory to analytically continue forth and back from Minkowski to Euclidean spaces in order to obtain a meaningfully regularized energy functional. In the present section we will discuss the consequences of this continuation on the eigenvalues and -vectors of the static Dirac Hamiltonian $h_{E}$ which can be extracted from eqn (1.11).

As inferred from eqn (1.5) the time components of the (axial) vector meson play the key role for the study of the analytic properties. The ansätze for the static meson field configurations are characterized by the fact that they commute with the grand spin operator $\boldsymbol{G}$. This operator denotes the sum of total angular momentum (orbital plus spin) and isospin, i.e. $\boldsymbol{G}=\boldsymbol{l}+\boldsymbol{\sigma} / 2+\boldsymbol{\tau} / 2$. Demanding in addition the proper behavior under parity the time component of the axial vector has to be zero while that of the vector field reduces to purely radial function $\omega(r)$. In Euclidean space the Dirac Hamiltonian may then be decomposed as

$$
h_{E}=h_{\Theta}+i \omega(r) \text {. }
$$

Here $h_{\Theta}$ denotes the Hermitian part of $h_{E}$, i.e. $h_{\Theta}^{\dagger}=h_{\Theta}$. Let us, for the time being, consider the simplest case when only pseudoscalar fields are present. The corresponding field configuration is assumed to be of hedgehog type

$$
\Sigma=m \exp (i \hat{\boldsymbol{r}} \cdot \boldsymbol{\tau} \Theta(r)) .
$$

Hence

$$
h_{\Theta}=\boldsymbol{\alpha} \cdot \boldsymbol{p}+m \beta\left(\cos \Theta+i \gamma_{5} \hat{\boldsymbol{r}} \cdot \boldsymbol{\tau} \sin \Theta\right) .
$$

Later on we will also consider the case when $\rho$ and $a_{1}$ fields are present as well. However, for the general discussion of the analytical properties the restriction to $\Theta$ and $\omega$ proves to be illuminating. For this discussion we generalize the Dirac Hamiltonian to an operator which depends on the complex variable $z$

$$
h(z)=h_{\Theta}+z \omega .
$$


The Euclidean space Hamiltonian, $h_{E}$ then corresponds to $z=i$ while the one in Minkowski space is associated with $z=1$. We will refer to these values of $z$ as Euclidean and Minkowski points, respectively. Except for the Minkowski point as well as $z=-1, h(z)$ is non-Hermitian. In general we therefore have to distinguish between left $\left(\Psi_{\nu}(z)\right)$ and right $\left(\tilde{\Psi}_{\nu}(z)\right)$ eigenstates

$$
\begin{aligned}
& h(z)\left|\Psi_{\nu}(z)\right\rangle=\epsilon_{\nu}(z)\left|\Psi_{\nu}(z)\right\rangle \\
& \left\langle\tilde{\Psi}_{\nu}(z)\left|h=\epsilon_{\nu}(z)\left\langle\tilde{\Psi}_{\nu}(z)\left|\quad \Longrightarrow \quad h^{\dagger}(z)\right| \tilde{\Psi}_{\nu}(z)\right\rangle=\epsilon_{\nu}(z)^{*}\right| \tilde{\Psi}_{\nu}(z)\right\rangle \text {. }
\end{aligned}
$$

Of course, the eigenvalues, $\epsilon_{\nu}(z)$, and -vectors, $\left|\Psi_{\nu}(z)\right\rangle$, parametrically depend on $z$. Adopting the set of free spherical grand spinors as basis [25] the matrix elements of $h_{\Theta}$ and $\omega$ turn out to be symmetric. Since $h^{\dagger}(z)=h\left(z^{*}\right)$ the eigenvalues and their complex conjugate are related via

$$
\epsilon_{\nu}(z)^{*}=\epsilon_{\nu}\left(z^{*}\right)
$$

Furthermore a phase convention may by chosen such that the wave-functions are related by

$$
|\tilde{\Psi}(z)\rangle=\left|\Psi(z)^{*}\right\rangle=\left|\Psi\left(z^{*}\right)\right\rangle .
$$

For later discussions it will be useful to define real and imaginary parts of the eigenvalues

$$
\epsilon_{\nu}^{R}\left(z, z^{*}\right)=\frac{1}{2}\left(\epsilon_{\nu}(z)+\epsilon_{\nu}\left(z^{*}\right)\right) \quad \epsilon_{\nu}^{I}\left(z, z^{*}\right)=-\frac{i}{2}\left(\epsilon_{\nu}(z)-\epsilon_{\nu}\left(z^{*}\right)\right)
$$

It is the main issue of this section to explore the $z$-dependence of the eigenvalues and -vectors once profile functions $\Theta(r)$ and $\omega(r)$ are made available. Of course, these test profiles can be chosen arbitrarily, however, they should (at least) be physically motivated. For convenience we will employ the profile functions displayed in figure 5.2 and constrain ourselves to the case $m=400 \mathrm{MeV}$. The reader may consult section 5 on the origin of these profiles. Here it suffices to remark that the $\omega$ field satisfies an equation analogous to (1.1) and is thus properly normalized. In order to study the behavior of the eigenvalues (and -vectors) in the complex plane defined by $z$ we parametrize

$$
z=\delta \exp (i \varphi) \quad \text { with } \quad 0 \leq \varphi \leq 2 \pi
$$

Obviously $\delta=1$ and $0 \leq \varphi \leq \pi / 2$ parametrizes the continuation from Minkowski to Euclidean space and vice versa. However, we will consider $\delta$ as a parameter in order to determine the radius of convergence in the complex $z$-plane. A priori it is not obvious that the energy eigenvalues exhibit any kind of analytic structure as these are roots of the characteristic polynomial (See e.g. appendix C of ref. 24].). This polynomial is in principle of infinite degree and only the restriction to a finite basis renders this degree finite as well. In figure 2.1 the dependence on $\delta$ for two different energy eigenvalues is shown. On the left the energy of the valence quark orbit is displayed. This orbit is, by definition, the one with the smallest absolute value of the real part of the energy eigenvalue. I.e. the valence quark state is associated with the smallest $\left|\epsilon_{\nu}^{R}\right|$ (see also section 3). The valence quark state is distinct since in the presence of static meson fields it is the one which deviates most strongly from a free quark state. Obviously the valence quark energy represents a smooth function of $\varphi$ for $\delta \leq 1$ while for $\delta=2$ the derivative $\partial \epsilon_{\text {val }} / \partial \varphi$ becomes singular. 
At the "edges" $\varphi$ assumes the values $\pm 0.32 \pi$. These singularities are, however, not the consequence of a non-analytic behavior of the roots of the characteristic polynomial but rather a level crossing appears. For $|\varphi| \leq 0.32 \pi$ a state with negligible $\varphi$-dependence carries the smallest $\left|\epsilon_{\nu}^{R}\right|$. For $|\varphi|>0.32 \pi$ the role of the valence quark is then taken over by an orbit which strongly depends on $\varphi$. Numerically we have confirmed that for the test profiles under consideration such level crossings in the valence quark channel are avoided as long as $\delta<1.3$. This radius of convergence suggests that for the relevant parameter space $(\delta=1)$ the eigenvalues of the Dirac Hamiltonian are analytic functions of $z$. This result was already obtained previously [26]. This is furthermore supported by considering other levels than the valence quark state. These actually possess a less pronounced dependence on $\omega$ and have thus a larger radius of convergence. As an example the energy of the state with the smallest $\left|\epsilon^{R}\right|$ in the channel with grand spin and parity $G^{\pi}=2^{+}$is displayed in figure 2.1. For this energy the radius of convergence is obviously larger than two.

There is actually a much more elegant technique to investigate the analytical structure of the eigenvalues and especially to extract the radius of convergence. As these eigenvalues are supposedly analytic in $z$ the corresponding Laurent expansion

$$
\epsilon_{\nu}(z)=\sum_{n=-\infty}^{\infty} c_{\nu, n}\left(z_{0}\right)\left(z-z_{0}\right)^{n}
$$

should exist. Here $z_{0}$ serves as the center of the expansion and may refer to any point in the complex plane. The Laurent coefficients $c_{\nu, n}\left(z_{0}\right)$ are defined by the Cauchy integrals

$$
c_{\nu, n}\left(z_{0}\right)=\frac{1}{2 \pi i} \oint_{\partial A} \frac{\epsilon_{\nu}(\zeta) d \zeta}{\left(\zeta-z_{0}\right)^{n+1}}
$$

with $z_{0}$ being in the interior of the integration contour $\partial A$, i.e. $z_{0} \in A$. Employing the parametrization $\zeta=\delta e^{i \varphi}+z_{0}$ these Cauchy integrals become ordinary integrals

$$
c_{\nu, n}\left(z_{0}\right)=\frac{1}{2 \pi \delta^{n}} \int_{0}^{2 \pi} \epsilon_{\nu}\left(\delta e^{i \varphi}+z_{0}\right) \exp (-i n \varphi) d \varphi .
$$

The computation of the integrals (2.12) obviously requires the eigenvalues of

$$
h_{\Theta}+\left(\delta e^{i \varphi}+z_{0}\right) \omega
$$

in the interval $0 \leq \varphi \leq 2 \pi$. Analyticity is then equivalent to the fact that the coefficients $c_{\nu, n}\left(z_{0}\right)$ do actually not depend on $\delta$. On the other hand the $c_{\nu, n}\left(z_{0}\right)$ are useful tools to determine the radius of convergence at $z_{0}$. For this purpose we start off with a small value for $\delta$, numerically compute the eigenvalues of (2.13) and subsequently the integrals (2.12). Next the calculations are repeated assuming a somewhat larger value for $\delta$. As long as $\delta$ is sufficiently small the $c_{\nu, n}\left(z_{0}\right)$ are indeed independent of $\delta$. However, as a critical value is exceeded the $c_{\nu, n}\left(z_{0}\right)$ vary with $\delta$. This critical value has to be identified as the radius of convergence at the point $z_{0}$. For the valence quark orbit the results of this calculation are displayed in table 2.1. Again we have employed the test profiles which have been mentioned above. For the interpretation of this table (and similar once which will follow) it should be stressed that the entry " 0.00 " means that this value is zero up to the given accuracy. On the other hand the entry "0" implies that the corresponding coefficient vanishes identically up to numerical uncertainties which usually are of the order $10^{-8}$. 
Table 2.1: The coefficients $c_{\mathrm{val}, n}\left(z_{0}\right)$ in the Laurent series (2.10) for the valence quark energy in units of the constituent quark mass.

\begin{tabular}{|c|cccc|cccc|}
\hline & \multicolumn{5}{|c|}{$z_{0}=0$} & \multicolumn{5}{c|}{$z_{0}=i$} \\
$\delta$ & 0.5 & 1.0 & 1.2 & 1.5 & 0.5 & 1.0 & 1.2 & 1.5 \\
\hline$n=-4$ & 0 & 0 & 0 & 0.18 & 0 & 0 & $0.08-0.07 i$ & $0.22+0.05 i$ \\
$n=-3$ & 0 & 0 & 0 & 0.14 & 0 & 0 & $0.06-0.09 i$ & $0.20-0.12 i$ \\
$n=-2$ & 0 & 0 & 0 & 0.08 & 0 & 0 & $0.04-0.10 i$ & $0.12-0.20 i$ \\
$n=-1$ & 0 & 0 & 0 & 0.02 & 0 & 0 & $0.01-0.10 i$ & $0.02-0.20 i$ \\
$n=0$ & 0.38 & 0.38 & 0.38 & 0.36 & $0.38+0.61 i$ & $0.38+0.61 i$ & $0.39+0.52 i$ & $0.36+0.46 i$ \\
$n=1$ & 0.59 & 0.59 & 0.59 & 0.56 & $0.62-0.03 i$ & $0.62-0.03 i$ & $0.60-0.09 i$ & $0.56-0.11 i$ \\
$n=2$ & -0.02 & -0.02 & -0.02 & -0.05 & $-0.01-0.02 i$ & $-0.01-0.02 i$ & $-0.03-0.07 i$ & $-0.06-0.05 i$ \\
$n=3$ & -0.01 & -0.01 & -0.01 & -0.03 & 0.00 & 0.00 & $-0.03-0.03 i$ & $-0.03-0.01 i$ \\
$n=4$ & 0.00 & 0.00 & 0.00 & -0.01 & 0.00 & 0.00 & $-0.02-0.02 i$ & -0.01 \\
\hline
\end{tabular}

Table 2.2: Same as table 2.1 for the state with the smallest (positive) real part of the energy eigenvalue in the $G^{\pi}=2^{+}$channel. All those coefficients which are not displayed either vanish $(n<0)$ or are negligibly small $(n \geq 2)$.

\begin{tabular}{|c|ccc|ccc|}
\hline & \multicolumn{3}{|c|}{$z_{0}=0$} & \multicolumn{3}{c|}{$z_{0}=i$} \\
$\delta$ & 0.5 & 1.0 & 1.5 & 0.5 & 1.0 & 1.5 \\
\hline$n=0$ & 1.1449 & 1.1449 & 1.1449 & $1.1453+0.0021 i$ & $1.1453+0.0021 i$ & $1.1453+0.0021 i$ \\
$n=1$ & 0.0022 & 0.0022 & 0.0022 & $0.0020-0.0005 i$ & $0.0020-0.0005 i$ & $0.0020-0.0005 i$ \\
\hline
\end{tabular}

Evaluating the Laurent coefficients associated with an expansion around the origin of the complex plane we conclude that the radius of convergence for the energy of the valence quark energy eigenvalue lies in between 1.2 and 1.5. In the region of convergence the Laurent series actually reduces to a Taylor series without any poles. Then the analytic continuation from Euclidean to Minkowski space is unique for this eigenvalue. However, as will be discussed in the next section, the physical relevant situation is somewhat different. The fermion determinant is defined in Euclidean space only. Thus one has to perform a Laurent expansion around $z_{0}=i$. The results for the coefficients stemming from this calculation are also shown in table 2.1 and demonstrate that for this expansion the radius of convergence is less than 1.2. Taking into account that the distance between the Euclidean and Minkowski points is $\sqrt{2}$ we see that it is not possible to carry out the required continuation for the valence quark level, at least for the test profiles considered here. Again this non-analyticity originates from a level crossing as discussed above. Thus the identification of the valence quark level does not necessarily symbolize an analytic operation. For orbits other than the valence quark the continuation is less problematic because their response to the $\omega$-profile is significantly weaker. As an example we have again considered the lowest state in the $2^{+}$channel. As can be inferred from table 2.2 the dependence of the corresponding energy eigenvalue on $z$ is almost negligible.

There is one more conclusion which can be drawn from the results listed in tables 2.1 and 2.2. In the region of convergence only those Laurent coefficients are sizable which correspond to a constant or linear dependence of the eigenvalues on $z$. In addition a small but non-vanishing quadratic piece may be present. This observation may be 
translated into the statement that the functional dependence of the energy eigenvalues on $\omega$ is at most quadratic. Of course, this again depends on the test profiles. However, our computations indicate that this is a generic feature for $\omega$-meson profiles which are in some way related to the quark baryon current and hence are properly normalized.

We have performed similar investigations not only for the eigenvalues but also for the eigenstates of $h(z)$ (see eqn (2.5)). The symmetry of $h_{\Theta}$ and $\omega$ in the standard basis straightforwardly relates left and right eigenstates by complex conjugation. It is thus sufficient to only consider the behavior of the right eigenstates in the complex $z$ plane. For the valence quark wave-function we display the dependence on $z=\delta \exp (i \varphi)$ in figure 2.2. Again we observe that the dependence is analytical for $\delta \leq 1.0$ and that for $\delta$ as large as 2.0 the identification of the valence quark level exhibits an arbitrariness. Although the somewhat shaky curve for the lower component seems to suggest some kind of non-analyticity even for $\delta=1.0$ this is actually not the case as we have verified by computing the Laurent series in analogy to (2.10).

At the end of this section we thus arrive at the statement that except for the identification of the valence quark state the diagonalization of $h(z)$ results in analytic expressions for its eigenvalues and -functions. For the identification of the valence quark state the radius of convergence is comparable to the distance between Euclidean and Minkowski points and depends on the center of the Laurent expansion. The identification will not cause problems if the self-consistent $\omega$-meson profiles turn out to be less pronounced than the test profiles considered here. Having computed a self-consistent solution it will be necessary to verify that the corresponding profile functions do not cause any level crossing for the valence quark state and the associated non-analytic behavior is avoided on the path from Euclidean to Minkowski spaces. Otherwise the solution has to discarded. 


\section{Analytic "Properties" of the Energy Functional}

In the previous section we have examined the analytic properties of the eigenvalues and -states of the one particle Dirac Hamiltonian (2.4) in the complex $z$-plane. Here we will investigate how (or whether at all) these properties propagate to the energy functional. For this purpose we will briefly review the derivation of the energy functional in Euclidean space and also emphasize on an important issue when evaluating the imaginary part of the action, $\mathcal{A}_{I}$, see eqn (1.8).

Ignoring for the moment the regularization of the fermion determinant associated with the Hamiltonian (2.4) it can formally be written as [27, 28]

$$
\operatorname{Det}\left(\not D_{E}\right)=\mathcal{C}(T) \exp \left(-T E_{0}\right) \sum_{\left\{\eta_{\nu}\right\}} \exp \left(-T N_{C} \sum_{\nu} \eta_{\nu} \bar{\epsilon}_{\nu}\right)
$$

Here $T$ denotes the Euclidean time interval under consideration. $\mathcal{C}(T)$ refers to a constant of proportionality which does not depend on the eigenvalues $\epsilon_{\nu}$. Hence its explicit form is of no further relevance for our present investigations. The sum goes over all possible configurations $\left\{\eta_{\nu}\right\}$ of (anti) quark occupation numbers $\eta_{\nu}=0,1$. The quantities $\bar{\epsilon}_{\nu}$ are defined in terms of the real and imaginary parts of the eigenvalues of $\epsilon_{\nu}$ (see eqn (2.8))

$$
\bar{\epsilon}_{\nu}=\left|\epsilon_{\nu}^{R}\right|+i \operatorname{sgn}\left(\epsilon_{\nu}^{R}\right) \epsilon_{\nu}^{I}=\operatorname{sgn}\left(\epsilon_{\nu}^{R}\right) \epsilon_{\nu}
$$

The limit $T \rightarrow \infty$ projects out the vacuum energy

$$
E_{0}=-\frac{N_{C}}{2} \sum_{\nu} \bar{\epsilon}_{\nu}
$$

from the exponential. Eqn. (3.1) provides a natural definition of the valence quark part of the energy

$$
E_{V}=E_{V}^{R}+i E_{V}^{I}=N_{C} \sum_{\nu} \eta_{\nu}\left|\epsilon_{\nu}^{R}\right|+i N_{C} \sum_{\nu} \eta_{\nu} \operatorname{sgn}\left(\epsilon_{\nu}^{R}\right) \epsilon_{\nu}^{I}
$$

It should be noted that the unregularized fermion determinant (3.1) actually is a function of the energy eigenvalues $\epsilon_{\nu}=\epsilon_{\nu}^{R}+i \epsilon_{\nu}^{I}$ rather than of their real and imaginary parts separately. Thus the complete fermion determinant (i.e. when the sum over all sets of occupation numbers is carried out) would represent an analytic function of $z$ if it were finite and as long as the eigenvalues were analytic functions of $z$. For practical calculations, however, one considers only configurations with a definite baryon number

$$
B=\sum_{\nu}\left(\eta_{\nu}-\frac{1}{2}\right) \operatorname{sgn}\left(\epsilon_{\nu}^{R}\right)
$$

This corresponds to restrict oneself to a special set of occupation numbers without carrying out the associated sum. Then an explicit dependence on a subset of the quantities $\bar{\epsilon}_{\nu}$ will induce a non-analyticity if $\operatorname{sgn}\left(\epsilon_{\nu}^{R}(z)\right)=\operatorname{sgn}\left(\epsilon_{\nu}(z)+\epsilon_{\nu}\left(z^{*}\right)\right)$ changes along the path connecting Euclidean and Minkowski spaces. From the discussion in the previous section it should be clear that such a change is most likely to happen for the valence quark orbit. All other states possess a sizable $\left|\epsilon_{\nu}^{R}\right|$ which is only slightly affected by the $\omega$-meson. Let us for the moment consider the case with baryon number $B=1$. The corresponding field configurations usually admit one distinct quark level which is referred 
to as the valence quark orbit. The static energy corresponding to a configuration where this state is occupied is hence given by

$$
\begin{aligned}
E & =E_{0}+N_{C} \eta_{\mathrm{val}} \bar{\epsilon}_{\mathrm{val}} \\
& =-\frac{N_{C}}{2} \sum_{\nu \neq \text { val }} \bar{\epsilon}_{\nu}+N_{C}\left(\eta_{\mathrm{val}}-\frac{1}{2}\right) \bar{\epsilon}_{\mathrm{val}} .
\end{aligned}
$$

As already mentioned above, the states other than the valence state are almost unaffected by the $\omega$-field. In particular only $\operatorname{sgn}\left(\epsilon_{\text {val }}^{R}\right)$ might vary when performing the analytic continuation. According to (3.5) the restriction to $B=1$ demands for the valence quark occupation number

$$
\eta_{\mathrm{val}}=\frac{1}{2}\left(1+\operatorname{sgn}\left(\epsilon_{\mathrm{val}}^{R}\right)\right)
$$

Substituting this result into eqn (3.7) yields

$$
E=\frac{N_{C}}{2}\left(\epsilon_{\mathrm{val}}-\sum_{\nu \neq \mathrm{val}} \bar{\epsilon}_{\nu}\right)
$$

which turns out to be analytic in $z$ in account on the above assertions on $\operatorname{sgn}\left(\epsilon_{\nu \neq \text { val }}^{R}\right)$.

The situation gets more involved when the fermion determinant is regularized. As already mentioned in the introduction the proper-time regularization requires to treat real and imaginary parts (1.8) separately. This leads to distinct treatments of $\epsilon_{\nu}(z)$ and $\epsilon_{\nu}(z)^{*}$. In turn the energy functional will depend on $z$ and its complex conjugate, i.e. $E=E\left(z, z^{*}\right)$. As only the vacuum part $E_{0}$ undergoes regularization the transition from eqn (3.6) to eqn (3.7) is, in general, prohibited. Thus there are (at least) two points which may cause the regularized energy functional to be non-analytic in $z$. Let us proceed step by step in deriving the regularized energy functional to see how this comes about.

At this point we wish to apply the proper-time regularization scheme to the real part of the fermion determinant

$$
\mathcal{A}_{R}=\frac{1}{2}\left\{\operatorname{Tr} \log \left(-\partial_{\tau}+h(z)\right)+\operatorname{Tr} \log \left(\partial_{\tau}+h^{\dagger}(z)\right)\right\}
$$

The functional trace is carried out by summing over the eigenvalues* $\Omega_{n}$ and $\epsilon_{\nu}(z)$ corresponding to the operators $\partial_{\tau}$ and $h(z)$, respectively. As we intent to extract $E_{0}^{R}$ from the expression (3.10) we need to consider the limit $T \rightarrow \infty$. Then the sum over $\Omega_{n}$ becomes a spectral integral

$$
\begin{aligned}
\mathcal{A}_{R} & =\frac{N_{C}}{2} T \int_{-\infty}^{\infty} \frac{d v}{2 \pi} \sum_{\nu}\left\{\log \left(i v+\epsilon_{\nu}\right)+\log \left(-i v+\epsilon_{\nu}^{*}\right)\right\} \\
& =\frac{N_{C}}{2} T \int_{-\infty}^{\infty} \frac{d v}{2 \pi} \sum_{\nu} \log \left\{\left|i v+\epsilon_{\nu}\right|^{2}\right\} .
\end{aligned}
$$

To this expression the proper-time prescription may be applied because the argument of the logarithm is positive definite

$$
\mathcal{A}_{R}=-\frac{N_{C}}{2} T \int_{-\infty}^{\infty} \frac{d v}{2 \pi} \sum_{\nu} \int_{1 / \Lambda^{2}}^{\infty} \frac{d s}{s} \exp \left\{-s\left[v^{2}+\left|\epsilon_{\nu}(z)\right|^{2}+2 v \epsilon_{\nu}^{I}(z)\right]\right\}
$$

${ }^{*} \Omega_{n}=(2 n+1) \pi / T$ are the Matsubara frequencies. The fermionic character of the quarks requires the associated eigenfunctions to assume anti-periodic boundary conditions on the Euclidean time interval $T$. 
Here it has to be stressed that this spectral integral cannot be obtained from the original definition of the proper-time regularization for the real part (1.9). Substituting (1.11) and (2.4) yields for (1.9)

$$
\mathcal{A}_{R}=-\frac{1}{2} \operatorname{Tr} \int_{1 / \Lambda^{2}}^{\infty} \frac{d s}{s} \exp \left\{-s\left[-\partial_{\tau}^{2}+h(z) h^{\dagger}(z)+\left(h(z)-h^{\dagger}(z)\right) \partial_{\tau}\right]\right\}
$$

because $\left[\partial_{\tau}, h(z)\right]=0$. The spectral integral (3.12) could only be obtained from (3.13) under the assumption that $h$ and $h^{\dagger}$ can be diagonalized simultaneously. At the Euclidean point this assumption is violated at quadratic order in $\omega_{4}$. Technically the difference arises from the fact that the usual rules for the logarithm have been used in order to derive (3.11). However, the original definition (1.9) of the proper-time regularization of $\mathcal{A}_{R}$ does not maintain these rules for finite $\Lambda$ because the regularization curbs the Hilbert space. Stated otherwise: Different regularization prescriptions induce errors in the energy functional at order $\omega_{4}^{2}$. It should furthermore be stressed that in deriving eqn (3.11) one has treated infinite quantities. This is avoided when the proper-time prescription is imposed at the operator level (1.9, 3.13). In section 5 we will argue from the point of the numerical results that (3.13) represents the more appropriate starting point. Nevertheless let us continue with the investigation of the expression (3.12) in particular to later on compare the various approaches for treating the $\omega$-meson in the NJL model.

This spectral integral (3.12) converges absolutely permitting in particular to shift the integration variable $v \rightarrow v-\epsilon_{\nu}^{I}(z)$

$$
\mathcal{A}_{R}=-\frac{N_{C}}{2} T \int_{-\infty}^{\infty} \frac{d v}{2 \pi} \sum_{\nu} \int_{1 / \Lambda^{2}}^{\infty} \frac{d s}{s} \exp \left\{-s\left[v^{2}+\left(\epsilon_{\nu}^{R}(z)\right)^{2}\right]\right\}
$$

Performing the Gaussian integral allows one to extract $\left(\mathcal{A}_{R} \rightarrow-T E_{0}^{R}\right)$

$$
E_{0}^{R}\left(z, z^{*}\right)=\frac{N_{C}}{2} \sum_{\nu} \int_{1 / \Lambda^{2}}^{\infty} \frac{d s}{\sqrt{4 \pi s^{3}}} \exp \left\{-s\left[\frac{\epsilon_{\nu}(z)+\epsilon_{\nu}\left(z^{*}\right)}{2}\right]^{2}\right\}
$$

which obviously depends on $z$ and its complex conjugate. In terms of the eigenvalues $\Omega_{n}$ and $\epsilon_{\nu}(z)$ the imaginary part of the action (1.8) reads

$$
\mathcal{A}_{I}=\frac{N_{C}}{2} \sum_{n, \nu} \log \frac{i \Omega_{n}-\epsilon_{\nu}(z)}{i \Omega_{n}-\epsilon_{\nu}\left(z^{*}\right)} .
$$

Now it is important to note that the associated spectral integral

$$
\int_{-\infty}^{\infty} \frac{d v}{2 \pi} \log \frac{i v-\epsilon_{\nu}(z)}{i v-\epsilon_{\nu}\left(z^{*}\right)}
$$

is not absolutely convergent. If it were one could easily eliminate the imaginary parts $\epsilon_{\nu}^{I}\left(z, z^{*}\right)$ of the energy eigenvalues from the integral (3.17) by reversing the sign of $v$ in the denominator and subsequently shifting $v$ by $\epsilon_{\nu}^{I}\left(z, z^{*}\right)$. We therefore need an extended definition of $\mathcal{A}_{I}$. We will later on see that the principle value prescription

$$
\mathcal{A}_{I}=\frac{N_{C}}{2} T \sum_{\nu} \mathcal{P} \int_{-\infty}^{\infty} \frac{d v}{2 \pi} \log \frac{i v-\epsilon_{\nu}(z)}{i v-\epsilon_{\nu}\left(z^{*}\right)}
$$


yields the desired physical symmetries such as the current field identity. The integral (3.18) can now be performed using standard means

$$
\begin{aligned}
\mathcal{A}_{I} & =\frac{-i N_{C}}{2} \sum_{\nu} T \mathcal{P} \int_{-\infty}^{\infty} \frac{d v}{2 \pi} \int_{-1}^{1} d \lambda \frac{\epsilon_{\nu}^{I}}{i\left(v-\lambda \epsilon_{\nu}^{I}\right)-\epsilon_{\nu}^{R}} \\
& =\frac{-i N_{C}}{2} \sum_{\nu} T \lim _{\mathcal{M} \rightarrow \infty} \int_{-\mathcal{M}}^{\mathcal{M}} \frac{d v}{2 \pi} \int_{-1}^{1} d \lambda \frac{\epsilon_{\nu}^{I}}{i\left(v-\lambda \epsilon_{\nu}^{I}\right)-\epsilon_{\nu}^{R}} .
\end{aligned}
$$

Next the shift in the integration variable $v-\lambda \epsilon_{\nu}^{I} \rightarrow v$ is performed. It can be shown that the associated shift in the boundaries does not contribute as $\mathcal{M} \rightarrow \infty$. This yields

$$
\mathcal{A}_{I}=\frac{-i N_{C}}{2} \sum_{\nu} \int_{-1}^{1} d \lambda T \lim _{\mathcal{M} \rightarrow \infty} \int_{-\mathcal{M}}^{\mathcal{M}} \frac{d v}{2 \pi} \frac{\epsilon_{\nu}^{I}}{i v-\epsilon_{\nu}^{R}}
$$

Now the integral over the parameter $\lambda$ may be done. Although $\mathcal{A}_{I}$ is finite in the principle value formulation, the proper time regularization may be imposed by expressing the integrand as a parameter integral:

$$
\frac{1}{v^{2}+\left(\epsilon_{\nu}^{R}\right)^{2}} \rightarrow \int_{1 / \Lambda^{2}}^{\infty} d s \exp \left\{-s\left(v^{2}+\left(\epsilon_{\nu}^{R}\right)^{2}\right)\right\}
$$

which does obviously not diverge as $\Lambda \rightarrow \infty$. We may finally extract the imaginary part of the Euclidean energy $E_{0}^{I}$ from $\mathcal{A}_{I} \rightarrow-i T E_{0}^{I}$

$$
\begin{aligned}
E_{0}^{I}\left(z, z^{*}\right)=\frac{i N_{C}}{4} \sum_{\nu}\left(\epsilon_{\nu}(z)-\epsilon_{\nu}\left(z^{*}\right)\right) \operatorname{sgn}\left(\epsilon_{\nu}(z)+\epsilon_{\nu}\left(z^{*}\right)\right) \\
\times\left\{\begin{array}{lll}
1, & \mathcal{A}_{I} & \text { not regularized } \\
\mathcal{N}_{\nu}\left(z, z^{*}\right), & \mathcal{A}_{I} & \text { regularized }
\end{array}\right.
\end{aligned}
$$

where we again made explicit the dependence on the complex variable $z$ and its complex conjugate. Furthermore

$$
\mathcal{N}_{\nu}\left(z, z^{*}\right)=\frac{1}{\sqrt{\pi}} \Gamma\left(\frac{1}{2},\left(\frac{\epsilon_{\nu}(z)+\epsilon_{\nu}\left(z^{*}\right)}{2 \Lambda}\right)^{2}\right)=\operatorname{erfc}\left(\left|\frac{\epsilon_{\nu}(z)+\epsilon_{\nu}\left(z^{*}\right)}{2 \Lambda}\right|\right)
$$

are the vacuum "occupation numbers" in the proper time regularization scheme. The upper case in eqn (3.22) corresponds to the limit $\Lambda \rightarrow \infty$. Previously [23, 24] the principle value description was not stated explicitly. Fortunately, due to the $\lambda$ integration the asymmetries in the boundaries cancel. Thus the final result remains unchanged.

Analogously to the studies in section 2 we have then considered the total energy functional originating from the fermion determinant

$$
E_{\mathrm{det}}\left(z, z^{*}\right)=E_{0}^{R}\left(z, z^{*}\right)+i E_{0}^{I}\left(z, z^{*}\right)+N_{C} \eta_{\mathrm{val}} \bar{\epsilon}_{\mathrm{val}}\left(z, z^{*}\right)
$$

in the complex $z$-plane. The imaginary part $E_{0}^{I}$ is taken in its regularized form. The behavior of $E\left(z, z^{*}\right)$ corresponding to the parametrization (2.9) is displayed in figure 3.1. The cuts which occur for $\delta=2$ stem from level crossings as described in the previous section. Otherwise $E\left(z, z^{*}\right)$ exhibits a smooth behavior on the angle $\varphi$. Although the valence and sea parts are treated differently the above indicated non-analyticity (see the discussion starting at eqn $(\underline{3.6}))$ due to the change of $\operatorname{sgn}\left(\epsilon_{\mathrm{val}}^{R}\left(z, z^{*}\right)\right)$ is mitigated. This is 
Table 3.1: Coefficients $c_{\operatorname{det}, n}\left(z_{0}\right)$ in the Laurent series for the contribution of the fermion determinant to the total energy (3.26). These coefficients are measured in GeV. Also shown are the associated continuations to the Minkowski point $E_{\mathrm{M}}=\tilde{E}_{\mathrm{det}}(z=1)$ as obtained from the coefficients quoted.

\begin{tabular}{|c|cccc|cccc|}
\hline & \multicolumn{5}{|c|}{$z_{0}=0$} & \multicolumn{5}{c|}{$z_{0}=i$} \\
\hline & 0.1 & 1.0 & 1.2 & 1.5 & 0.1 & 1.0 & 1.2 & 1.5 \\
\hline$n=-4$ & 0.000 & -0.007 & 0.036 & -0.029 & 0.000 & -0.008 & $0.061-0.085 i$ & $0.031+0.043 i$ \\
$n=-3$ & 0.000 & -0.006 & -0.014 & 0.135 & 0.000 & $-0.004+0.032 i$ & $0.066-0.013 i$ & $0.207+0.245 i$ \\
$n=-2$ & 0.000 & -0.064 & -0.136 & -0.260 & 0.000 & $-0.045+0.012 i$ & $-0.051-0.098 i$ & $-0.111-0.192 i$ \\
$n=-1$ & 0.000 & -0.005 & 0.069 & 0.330 & $0.003 i$ & $0.015+0.312 i$ & $0.039+0.370 i$ & $0.058+0.636 i$ \\
$n=0$ & 1.208 & 1.348 & 1.425 & 1.572 & $0.706+0.655 i$ & $0.922+0.654 i$ & $1.024+0.552 i$ & $1.202+0.481 i$ \\
$n=1$ & 0.650 & 0.655 & 0.656 & 0.618 & $0.689+1.164 i$ & $0.675+1.155 i$ & $0.649+1.078 i$ & $0.602+1.069 i$ \\
$n=2$ & 0.740 & 0.742 & 0.744 & 0.717 & $0.484-0.024 i$ & $0.528-0.013 i$ & $0.512-0.068 i$ & $0.510-0.048 i$ \\
$n=3$ & -0.014 & -0.013 & -0.012 & -0.032 & $0.008+0.164 i$ & $0.004+0.124 i$ & $-0.028+0.088 i$ & $-0.033+0.111 i$ \\
$n=4$ & 0.042 & 0.041 & 0.039 & 0.028 & $0.075-0.054 i$ & $0.023-0.003 i$ & $-0.003-0.020 i$ & $0.009+0.004 i$ \\
\hline$E_{\mathrm{M}}(\mathrm{GeV})$ & 2.627 & 2.700 & 2.673 & 2.782 & $2.523+0.035 i$ & $2.712-0.036 i$ & $2.711+0.137 i$ & $2.715+0.029 i$ \\
\hline
\end{tabular}

a special feature of the proper-time regularization which contains the equivalence between the limits $\epsilon_{\nu}^{R} \rightarrow 0$ and $\Lambda \rightarrow \infty$ in eqns (3.15, 3.22). This is intuitively clear because these regulator functions only depend on the dimensionless ratios $\epsilon_{\nu}^{R} / \Lambda$. The smooth behavior of $E\left(z, z^{*}\right)$ in the complex $z$-plane for the physically relevant radius $\delta \approx 1$ suggests that $E\left(z, z^{*}\right)$ resembles an analytic function. This, of course, would be somewhat astonishing because $z^{*}$ cannot be expressed as a power series in $z$ without trading in an essential singularity. As $E\left(z, z^{*}\right)$ depends on both $z$ and $z^{*}$ this essential singularity should carry through to the energy functional. In order to clarify the analytic properties of the energy functional (3.24) we have also examined the corresponding Laurent series

$$
\tilde{E}_{\operatorname{det}}(z):=\sum_{n=-\infty}^{\infty} c_{\operatorname{det}, n}\left(z_{0}\right)\left(z-z_{0}\right)^{n} .
$$

Again we considered expansion centers located at the origin of the complex plane $\left(z_{0}=0\right)$ and the Euclidean point $\left(z_{0}=i\right)$. The results are summarized in table 3.1.

There are several observations to be made. First, we find non-vanishing coefficients $c_{\text {det }, n}$ with $n<0$ even for parametrizations which led to non-singular one-particle energies $\epsilon_{\nu}(z)$. Second, these coefficients obviously depend on the path $(\partial A)$ along which the Cauchy integrals

$$
c_{\mathrm{det}, n}\left(z_{0}\right)=\frac{1}{2 \pi i} \oint_{\partial A} d \zeta \frac{E_{\mathrm{det}}\left(\zeta, \zeta^{*}\right)}{\left(\zeta-z_{0}\right)^{n+1}}
$$

are computed. The path $\partial A$ is again parametrized by $\zeta=\delta \exp (i \varphi)$ with $(0 \leq \varphi \leq 2 \pi)$. Even for small areas $A$ which include the expansion center $\left(z_{0} \in A\right)$ the coefficients $c_{\operatorname{det}, n}$ do not converge. Especially the coefficient of the constant term $c_{\text {det }, 0}$ varies by about $20 \%$ when decreasing $\delta$ from unity to 0.1 . For both values of $\delta$ the one-particle energies were found to be analytic, see section 2. Furthermore we extract from our numerical studies that (for a given path $\partial A$ ) the coefficients $c_{\text {det, } n}$ decrease only very slowly as $|n|$ gets larger. Thus we have accumulated (at least) three arguments which support 
the conclusion that the Laurent series (3.25) does not converge: The coefficients $c_{\operatorname{det}, n}$ with $n<0$ do not vanish, the radius of convergence shrinks to zero, and the Minkowski extracted by analytic continuation has a non-vanishing imaginary part. We have then considered the continuation to the Minkowski point $E_{\mathrm{M}}=\tilde{E}_{\operatorname{det}}(z=1)$. Again we find a dependence on $\delta$. This dependence is somewhat weaker than in the case of the coefficients $c_{\operatorname{det}, n}$. We should, however, note that for the computation of $E_{\mathrm{M}}$ only those coefficients which are displayed in table 3.1 were taken into consideration; i.e. $|n| \leq 4$. The reason is that the numerical computation of the coefficients with larger $n$ contains significant errors for small $d i$. A further evidence for the non-existence of the series (3.25) can be deduced from the fact that $E_{M}$ is not real when the expansion is centered at the Euclidean point. We close our considerations on the structure of $E_{\text {det }}$ with the statement that the associated Laurent series has vanishing radius of convergence. Stated more drastically, the analytic continuation of $E_{\text {det }}$ does not exist. And in general

$$
E_{\operatorname{det}}\left(z, z^{*}\right) \not \equiv \tilde{E}_{\operatorname{det}}(z) \text {. }
$$

The Euclidean point resembles the only exception to this inequality.

The non-existence of an analytic continuation for $\delta \leq 1$ is purely caused by the proper-time regularization of the Euclidean action. As this regularization treats real and imaginary parts of the one-particle energies differently it is interesting to consider other regularization schemes which do not have this property. The most suggestive approach would be to regularize the vacuum energy (3.3) by employing a sharp cut-off and include all states with $\left|\epsilon_{\nu}^{R}\right| \leq \Lambda$ in the summation. We have also studied the analytic properties of an energy functional defined in this way. Indeed it yields a small but finite radius of convergence. In this area of convergence the coefficients $c_{\operatorname{det}, n}$ of negative index $n<0$ vanish. Convergence is lost as one of the real parts of the energy eigenvalues crosses $\pm \Lambda$ along the path connecting Euclidean and Minkowski space. Since numerically this radius of convergence is found to be significantly smaller than unity (which is the physically relevant one) we will not further pursue this treatment of the energy functional. Furthermore a sharp cut-off regularization should be discarded because it does not even preserve global gauge invariance.

\section{Consistency Conditions for the Energy Functional}

In the last section we have learned that no analytic continuation of the regularized energy functional exists. One therefore has to reside on physical arguments, as e.g. gauge symmetry, in order to define (or motivate) an energy functional in Minkowski space. This will be the contents of the first part of this section. Actually three distinct approaches to the Minkowski energy functional are known in the literature. Historically the first to consider the $\omega$ meson in the context of the NJL model were Watabe and Toki [20]. Secondly a different calculation performed by researchers at Bochum University [21, 22, while the third approach was put forward by the present authors [23, 24]. In the second part of the present section we will examine these approaches from two points of view: (1) their relation to the Euclidean energy functional and (2) their consistency with the conditions which will be derived in the first part of this section.

A constant shift of the $\omega$-field $\omega(x) \rightarrow \omega(x)+\omega_{c}$ is equivalent to a global gauge transformation and should leave the fermion determinant unchanged, at least for topologically trivial configurations. The non-regularized form of the fermion determinant

${ }^{\dagger}$ The $c_{\text {det }, n}$ 's are of the generic form $I \times \delta^{-n}, c f$. eqn (2.12). Thus numerical errors in the integral $I$ are multiplied for small $\delta$ and large $n$. 
(3.1) can easily be seen to possess this property. This is mostly a consequence of the principle value prescription for the imaginary part of the action (3.18). At the Euclidean point a constant shift in $\omega$ causes the imaginary part of the energy eigenvalues to change accordingly

$$
\epsilon_{\nu}^{I} \rightarrow \epsilon_{\nu}^{I}+\omega_{c} \quad \text { as } \quad \omega(x) \rightarrow \omega(x)+\omega_{c} .
$$

This straightforwardly transfers to that part of the energy which stems from the fermion determinant

$$
E_{0} \rightarrow E_{0}-i \frac{N_{C}}{2} \omega_{c} \sum_{\nu} \operatorname{sgn}\left(\epsilon_{\nu}^{R}\right) \quad \text { and } \quad E_{V} \rightarrow E_{V}+i N_{C} \omega_{c} \sum_{\nu} \eta_{\nu} \operatorname{sgn}\left(\epsilon_{\nu}^{R}\right)
$$

such that

$$
E_{\mathrm{det}} \rightarrow E_{\mathrm{det}}+i N_{C} B \omega_{c}
$$

This is just the desired result that shifting the $\omega$ field by a constant changes the energy (associated with the fermion determinant) by an amount proportional to the baryon number. This is not surprising since a constant $\omega_{c}$ represents a chemical potential for the quarks. Since the unregularized form of the fermion determinant has been seen to be analytic it is obvious that the relation (4.3) can be continued to Minkowski space. I.e. the Minkowski energy changes by $N_{C} B \omega_{c}$. We therefore require a suitable definition of the regularized energy functional in Minkowski space to transform accordingly under $\omega(x) \rightarrow \omega(x)+\omega_{c}$. At this point we also recognize the reason why a sharp cut-off regularization in Minkowski space does not meet this requirement. In case $\omega_{c}$ shifts one of the eigenvalues across this sharp cut-off this special state is dropped in the sum (3.3). Then the transformation property (4.3) is no longer valid. The same behavior can be observed for other regularization schemes, as e.g. a three dimensional momentum cutoff, which operate in Minkowski space. If one wanted to implement the transformation relation (4.3) for a three dimensional momentum cut-off in Minkowski space one would inevitably be led to a regularization function which depended on $\omega_{c}$. This feature is, of course, undesired. In Euclidean space, however, only $\epsilon_{\nu}^{R}$ enters the regularization condition. Thus the sharp cut-off or three momentum regularization in Euclidean space is consistent with (4.3) because the shift (4.1) only effects the imaginary parts $\epsilon_{\nu}^{I}$.

It should be remarked that the transformation property (4.3) holds in Skyrme type models as well. In those models the coupling between $\omega$ and the chiral field is of the form $N_{C} \omega_{\mu} B^{\mu}$ [30]. Shifting the $\omega$ field by a constant leads to the variation of the energyt

$$
N_{C} \int d^{3} r \omega_{c} B_{0}=N_{C} \omega_{c} \int d^{3} r B_{0}=N_{C} \omega_{c} B
$$

In order to extract the baryon current one introduces an external space-dependent gauge field $b_{\mu}(x)$ for the $U_{V}(1)$ symmetry. The baryon current $B_{\mu}$ is then identified as the coupling linear in $b_{\mu}(x)$, i.e.

$$
j_{I=0}^{\mu}=\left.\frac{\delta \mathcal{A}[b]}{\delta b_{\mu}(x)}\right|_{b_{\mu}(x)=0} .
$$

* Other couplings appear as total derivatives of topologically trival objects and thus do not contribute when shifting the $\omega$-field. 
The action $\mathcal{A}[b]$ is defined by replacing $V$ with $Y+\not b$ in eqn (1.7). This guarantees the local $U_{V}(1)$ symmetry. Under the functional integral the vector meson fields may be shifted by an arbitrary amount (again up to topological defects). This allows one to transfer the dependence on the gauge field to the mesonic part of the action (1.6) and straightforwardly obtain the baryon current

$$
j_{I=0}^{\mu} \propto \frac{1}{2 G_{2}} \operatorname{tr}\left(V^{\mu}\right)=\frac{1}{G_{2}} \omega^{\mu}
$$

i.e. the current field identity (1.2) holds. In chiral soliton models the identity (4.6) commonly is a consequence of the stationary condition for the $\omega$-field. This condition is obtained by extremizing the Minkowski energy functional. Thus we have at hand a second consistency condition for the Minkowski energy functional. For the static $\omega$-field this can also be interpreted as the normalization of the $\omega$-field

$$
\int d^{3} r \omega(r)=G_{2} B
$$

We will require the equation of motion for $\omega(r)$ to provide this normalization. This normalization also has the important consequence that the inclusion of the $\omega$ meson into the soliton calculation does not necessarily lower the static energy although the number of degrees of freedom is increased.

The first approach to incorporate the $\omega$ meson in the NJL soliton calculation was carried out by Watabe and Toki[20]. Their calculation was completely performed in Minkowski space. In this treatment the contribution of the fermion determinant to the energy functional has been defined as

$$
E_{\mathrm{det}}^{(1)}=E_{0}^{(1)}+E_{V}^{(1)}=\frac{N_{C}}{2} \sum_{\nu} \int_{1 / \Lambda^{2}}^{\infty} \frac{d s}{\sqrt{4 \pi s^{3}}} \exp \left(-s \epsilon_{\nu}^{2}\right)+N_{C} \eta_{\mathrm{val}} \epsilon_{\mathrm{val}}
$$

wherein $\epsilon_{\nu}$ denote the eigenvalues of $h(z=1)$ (2.4). Although this is formally the analytic continuation of (3.15) it has the disadvantage that the above stated consistency conditions are not satisfied. E.g. the shift $\omega(x) \rightarrow \omega(x)+\omega_{c}$ leads to $\epsilon_{\nu} \rightarrow \epsilon_{\nu}+\omega_{c}$ and thus all powers of $\omega_{c}$ appear in (4.8) after the shift. However, the requirement (4.3) only allows for a linear term. Furthermore as the derivativet

$$
\sum_{\mu} \frac{\partial E_{0}^{(1)}}{\partial \epsilon_{\mu}}=-\frac{N_{C}}{2} \sum_{\mu} \operatorname{sgn}\left(\epsilon_{\mu}\right) \operatorname{erfc}\left(\left|\frac{\epsilon_{\mu}}{\Lambda}\right|\right) \neq-\frac{N_{C}}{2} \sum_{\mu} \operatorname{sgn}\left(\epsilon_{\mu}\right)
$$

the normalization condition (4.7) is not satisfied. Since the analytic continuation of (3.24) does actually not exist it is not surprising that the formal continuation does not meet the consistency conditions. As these are not met by (4.8) we do not consider it as a suitable definition for the Minkowski energy functional.

Soon after this, Schüren et al. [21, 22] presented an approach which assumes as starting point the Euclidean action in the proper-time regularization scheme (3.15.3.22). In order to reach the Minkowski point these authors "interpreted" the eigenvalues $\epsilon_{\nu}^{ \pm}$of the operators $h(z= \pm 1)$ as the continuations of $\epsilon_{\nu}( \pm i)$. Then

$$
E_{\operatorname{det}}^{(2)}=\frac{N_{C}}{2} \sum_{\nu}\left\{\int_{1 / \Lambda^{2}}^{\infty} \frac{d s}{\sqrt{4 \pi s^{3}}} \exp \left(-\frac{s}{4}\left(\epsilon_{\nu}^{+}+\epsilon_{\nu}^{-}\right)^{2}\right)+\frac{1}{2}\left(\epsilon_{\nu}^{+}-\epsilon_{\nu}^{-}\right) \operatorname{sgn}\left(\epsilon_{\nu}^{+}+\epsilon_{\nu}^{-}\right)\right\}
$$

†This derivative can, however, be identified as the regularized baryon number carried by the polarized Dirac sea. 
was defined as the contribution of the Dirac sea to the energy functionat团. Taking analyticity of the one-particle energy eigenvalues for granted eqn (4.10) amounts to

$$
E_{\text {det }}^{(2)}=E_{\text {det }}(1,-1)
$$

Apparently this interpretation cannot be characterized as an analytical continuation. Advantageously the definition (4.10) allows one to identify certain powers of the $\omega$-field in the sence that $\epsilon_{\nu}^{+}$is even while $\epsilon_{\nu}^{-}$is odd in agreement with $\epsilon_{\nu}^{R}$ and $\epsilon_{\nu}^{I}$, respectively. However, already the quadratic order in $\omega$ (contained in $\epsilon_{\nu}^{+}$) of $E_{\text {det }}^{(2)}$ differs significantly from the expansion in $\omega_{4}$ which starts from the expression $\not D_{E} \not D_{E}^{\dagger}$. This subject will be discussed at length in section 5 . Here it is merely important to keep in mind that counting powers of $\omega$ in $\epsilon^{ \pm}$is (at least) suspicious; most likely faulty. In contrast to the work of Watabe and Toki [20] the treatment of Schüren et al. satisfies the above derived consistency conditions. Shifting the $\omega$-field by a constant implies $\epsilon_{\nu}^{-} \rightarrow \epsilon_{\nu}^{-}+\omega_{c}$ while $\epsilon_{\nu}^{+}$remains unchanged. Substituting this transformation prescription into eqn (4.10) immediately provides the analytical continuation of (4.3). The normalization condition (4.7) is verified by integrating eqn (4.37) of ref. 22, which represents the equation of motion in this approach, over the whole coordinate space.

The approach of the present authors to include the $\omega$ degree of freedom into the NJL soliton calculations is based on an observation made in section 2 . When investigating the analytic structure of the single quark eigenenergies it was found that in the physical relevant region the Laurent series centered at the Euclidean point contained only two significant terms, a constant and the expression linear in the complex variable $z$, see tables 2.1 and 2.2. Transferring this approximation to the total fermion determinant implies to identify

$$
E_{\operatorname{det}}(z) \approx E_{0}^{R}(i,-i)+z E_{0}^{I}(i,-i)
$$

because at the Euclidean point the real (imaginary) part contains only even (odd) terms in $\omega_{4}$. This approximation is furthermore supported by the fact that the ambiguities for regularizing $\mathcal{A}_{R}$ are of order $\omega_{4}^{2}$ (see the discussion proceeding eqn (3.13)). By construction, the approximation (4.12) represents an analytic function of $z$ which can be continued to Minkowski space yielding

$$
E_{\text {det }}^{(3)}=E_{0}^{R}(i,-i)+E_{0}^{I}(i,-i) .
$$

It should be stressed that in contrast to the two other approaches the evaluation of $E_{\text {det }}^{(3)}$ indeed requires the diagonalization of a non-Hermitian Dirac Hamiltonian. The oneparticle energies which are evaluated in Euclidean space enter this expression for the Minkowski energy functional whence it is clear that the condition (4.3) stemming from the global gauge invariance is satisfied. The normalization condition (4.7) is proven to hold by integrating the equation of motion for the $\omega$ profile function (Eqn (B.6) of ref. [24]) over the coordinate space .

To conclude this section we would like to make some remarks on a possible saddle point approximation to the functional integral in Euclidean space. In this approximation the stationary conditions for the profile functions are obtained by identifying the configuration

\footnotetext{
${ }^{\ddagger}$ The imaginary part (3.22) has been taken in the unregularized version.

§In ref.24 we have considered the imaginary part of the action in the regularized as well as in the unregularized form. The consistency conditions are only satisfied for the unregularized form.
} 
with the smallest real part of the Euclidean action. The reason is that this configuration is assumed to dominate the functional integral. The resulting equation of motion acquires the form

$$
0=\frac{\delta E_{R}(i,-i)}{\delta \varphi(r)}
$$

where $E_{R}(i,-i)=E_{0}^{R}(i,-i)+E_{m}(i)$ also includes a part which originates from the mesonic part of the action (1.6) continued to Euclidean space. Furthermore $\varphi$ denotes the meson field under consideration. As $E_{R}(i,-i)$ contains only even powers of $\omega$ the saddle point equation (4.14) possesses the undesired solution $\omega \equiv 0$. This solution does clearly not satisfy the global gauge symmetry condition (4.3). Thus the saddle point condition has to be amended in order to comply with the consistency conditions. Obviously the extremization of the functional in which the fermion determinant is replaced by the approximation (4.12) at $z=1$ represents a suitable extension of the saddle point equation. Although this replacement is not unique it provides further justification for our treatment of the $\omega$ meson.

\section{Time Components up to Quadratic Order}

Up to this point the situation looks somewhat discouraging because we have merely accumulated arguments why several approaches cannot be considered correct. These approaches started on a complete non-perturbative treatment of the $\omega$ meson in the NJL soliton calculation. We have seen that then an exact analytic continuation from Euclidean to Minkowski space is impossible for the regularized module of the fermion determinant. In this section we will thus return to a perturbative type of treatment for the $\omega$-field and investigate up to which extent one can make contact to the non-perturbative calculations discussed in the previous sections.

We start off with the Euclidean Dirac Hamiltonian $h_{\Theta}+i \omega_{4}$ and consider $\omega_{4}$ as a perturbation to $h_{\Theta}$. Standard perturbation techniques $\$$ may be employed for the energy eigenvalues to illustrate the $\omega_{4}$ dependence

$$
\epsilon_{\nu}=\epsilon_{\nu}^{0}+\epsilon_{\nu}^{1}+\epsilon_{\nu}^{2} \ldots
$$

with

$$
\epsilon_{\nu}^{1}=i\left\langle\nu\left|\omega_{4}\right| \nu\right\rangle \quad \text { and } \quad \epsilon_{\nu}^{2}=-\sum_{\mu \neq \nu} \frac{\left\langle\nu\left|\omega_{4}\right| \mu\right\rangle\left\langle\mu\left|\omega_{4}\right| \nu\right\rangle}{\epsilon_{\nu}^{0}-\epsilon_{\mu}^{0}}
$$

Here $\epsilon_{\nu}^{0}$ denote the eigenvalues of $h_{\Theta}$ and $|\nu\rangle$ the corresponding eigenstates. The distinction between real and imaginary parts is trivial

$$
\epsilon_{\nu}^{R}=\epsilon_{\nu}^{0}-\sum_{\mu \neq \nu} \frac{\left\langle\nu\left|\omega_{4}\right| \mu\right\rangle\left\langle\mu\left|\omega_{4}\right| \nu\right\rangle}{\epsilon_{\nu}^{0}-\epsilon_{\mu}^{0}} \quad \text { and } \quad \epsilon_{\nu}^{I}=i\left\langle\nu\left|\omega_{4}\right| \nu\right\rangle .
$$

In table 5.1 we summarize the numerical results of this perturbative calculation for the low-lying states in the $G^{\pi}=0^{+}$channel which also contains the valence quark level. Again we have adopted the standard reference profile functions already used to gain the

*These techniques assume a complete Hilbert space. However, regularization effectively reduces this space. 
Table 5.1: Perturbation for the one-particle energy eigenvalues of the complex Dirac Hamiltonian in the $G^{\pi}=0^{+}$channel. All numbers are in units of the constituent quark mass $m$.

\begin{tabular}{|ccc|c|c|}
\hline \multicolumn{4}{|c|}{ Perturbation } & Exact \\
$\epsilon_{\nu}^{0}$ & $\epsilon_{\nu}^{1}$ & $\epsilon_{\nu}^{2}$ & total & $h(z=i)$ \\
\hline-1.522 & $0.109 i$ & -0.016 & $-1.537+0.109 i$ & $-1.550+0.119 i$ \\
-1.293 & $0.093 i$ & -0.026 & $-1.319+0.093 i$ & $-1.333+0.086 i$ \\
-1.118 & $0.069 i$ & -0.042 & $-1.159+0.067 i$ & $-1.150+0.042 i$ \\
-1.029 & $0.018 i$ & -0.022 & $-1.051+0.018 i$ & $-1.037+0.069 i$ \\
0.382 & $0.595 i$ & 0.019 & $0.401+0.595 i$ & $0.397+0.605 i$ \\
1.071 & $0.011 i$ & 0.006 & $1.072+0.011 i$ & $1.072+0.009 i$ \\
1.246 & $0.034 i$ & 0.004 & $1.240+0.034 i$ & $1.250+0.029 i$ \\
1.481 & $0.056 i$ & 0.009 & $1.491+0.056 i$ & $1.490+0.050 i$ \\
\hline
\end{tabular}

results presented in sections 2 and 3. We observe that the perturbation series seems to converge quickly and that the second order $\left(\epsilon_{\nu}^{2}\right)$ is already negligibly small. Furthermore the comparison with the exact diagonalization of the Euclidean Dirac Hamiltonian shows reasonable agreement at the level of a few percent. We have observed a similar behavior for states not listed in table 5.1. This corroborates our earlier assumptions which led to the approximation (4.12).

It is then intuitive to transfer this expansion to the complete energy functional at the Euclidean point. This will then be an analytic function in $\omega_{4}$ which can straighforwardly be continued to Minkowski space via $\omega_{4} \rightarrow-i \omega_{0}$. Up to quadratic order in $\omega_{4}$ the energy functional in Euclidean space is given by

$$
\begin{array}{r}
E_{2}^{\text {Eucl }}\left[\Theta, \omega_{4}\right]=E_{0}[\Theta]+i N_{C} \eta_{\text {val }}\left\langle\text { val }\left|\omega_{4}\right| \text { val }\right\rangle-i \frac{N_{C}}{2} \sum_{\nu} \operatorname{sgn}\left(\epsilon_{\nu}^{0}\right) \operatorname{erfc}\left(\left|\frac{\epsilon_{\nu}^{0}}{\Lambda}\right|\right)\left\langle\nu\left|\omega_{4}\right| \nu\right\rangle(5.4) \\
-N_{C} \eta_{\text {val }} \sum_{\nu \neq \text { val }} \frac{\mid\left.\left\langle\nu\left|\omega_{4}\right| \text { val }\right\rangle\right|^{2}}{\epsilon_{\text {val }}^{0}-\epsilon_{\nu}^{0}}+\frac{N_{C}}{2} \sum_{\nu \mu} \tilde{f}\left(\epsilon_{\nu}^{0}, \epsilon_{\mu}^{0} ; \Lambda\right)\left|\left\langle\nu\left|\omega_{4}\right| \mu\right\rangle\right|^{2}+\frac{4 \pi}{3} \frac{m_{\omega}^{2} f_{\pi}^{2}}{m^{2}} \int d r r^{2} \omega_{4}^{2} .
\end{array}
$$

Here $E_{0}[\Theta]$ refers to the energy functional associated with the Hermitian part of the Dirac Hamiltonian $h_{\Theta}$. Also the subtraction for the trivial vacuum configuration $\Theta \equiv 0$ is contained in $E_{0}[\Theta]$. For the valence quark contributions (proportional to $\eta_{\text {val }}$ ) the application of (5.1) and (5.2) is straightforward. The corresponding calculation for the Dirac sea, which yields the regulator function

$$
\tilde{f}\left(\epsilon_{\nu}, \epsilon_{\mu} ; \Lambda\right)=\left\{\begin{array}{cl}
\frac{1}{2} \frac{\operatorname{sgn}\left(\epsilon_{\nu}\right) \operatorname{erfc}\left(\left|\frac{\epsilon_{\nu}}{\Lambda}\right|\right)-\operatorname{sgn}\left(\epsilon_{\mu}\right) \operatorname{erfc}\left(\left|\frac{\epsilon_{\mu}}{\Lambda}\right|\right)}{\epsilon_{\nu}-\epsilon_{\mu}}, & \epsilon_{\nu} \neq \epsilon_{\mu} \\
0, & \epsilon_{\nu}=\epsilon_{\mu}
\end{array}\right.
$$

may be found in the appendix. Here it is important to remark that this regulator function has vanishing diagonal elements $\left(\tilde{f}\left(\epsilon_{\nu}, \epsilon_{\nu} ; \Lambda\right)=0\right)$ as a consequence of the second order perturbation formula (5.2). However, the limit $\epsilon_{\mu} \rightarrow \epsilon_{\nu}$ is not assumed smoothly. The last term in eqn (5.4) stems from (1.6) with the gradient expansion result [15] $G_{2}=$ $3 m^{2} / 2 m_{\omega}^{2} f_{\pi}^{2}$ substitutedit. In case one wants to consider the model with the imaginary

\footnotetext{
${ }^{\dagger}$ Here we do not include the effect of $\pi-a_{1}$ mixing.
} 
Table 5.2: Perturbation for the energy corresponding to the fermion determinant at the Euclidean point. All numbers are in $\mathrm{MeV}$.

\begin{tabular}{|c|ccc|c|c|}
\hline & \multicolumn{4}{|c|}{ Perturbation } & Exact \\
& $0^{\text {th }}$ & $1^{\text {st }}$ & $2^{\text {nd }}$ & $E_{2}^{\text {Eucl }}$ & $E\left(z=i, z^{*}=-i\right)$ \\
\hline$E_{V}$ & 458 & $714 i$ & 19 & $477+714 i$ & $476+726 i$ \\
$E_{0}+E_{V}$ & 1261 & $681 i$ & -553 & $724+681 i$ & $705+692 i$ \\
\hline
\end{tabular}

part of the action not being regularized the complementary error function in eqn (5.4) has to be replaced by unity. In that case the consistency condition (4.3) is satisfied. This is easy to see since for a constant $\omega$-field $\left\langle\nu\left|\omega_{c}\right| \mu\right\rangle=\omega_{c} \delta_{\nu \mu}$. As already mentioned the diagonal elements do not contribute at quadratic and higher order hence the whole change under the constant shift is proportional to the baryon number. From the numerical results listed in table 5.2 we find that the expansion (5.4) is reliable at the 3 percent level although the contribution from the Dirac sea quadratic in $\omega_{4}$ is unexpectedly sizable.

Although these results are encouraging we will now argue that $\tilde{f}\left(\epsilon_{\nu}, \epsilon_{\mu} ; \Lambda\right)$ in eqn (5.5) is not the correct regularization function at quadratic order. We have already remarked the unpleasant feature that $\tilde{f}\left(\epsilon_{\nu}, \epsilon_{\mu} ; \Lambda\right)$ is discontinuous as $\epsilon_{\mu} \rightarrow \epsilon_{\nu}$. A second disadvantage of $\tilde{f}$ relates to the Minkowski energy and the resulting equation of motion for $\omega_{0}$

$$
\begin{aligned}
2 \int d r^{\prime} r^{\prime 2} \tilde{\mathcal{K}}\left(r, r^{\prime}\right) \omega_{0}\left(r^{\prime}\right)= & \eta_{\text {val }} \int d \Omega \Psi_{\text {val }}^{\dagger}(\boldsymbol{r}) \Psi_{\text {val }}(\boldsymbol{r}) \\
& -\frac{1}{2} \sum_{\nu} \operatorname{sgn}\left(\epsilon_{\nu}^{0}\right) \operatorname{erfc}\left(\left|\frac{\epsilon_{\nu}^{0}}{\Lambda}\right|\right) \int d \Omega \Psi_{\text {val }}^{\dagger}(\boldsymbol{r}) \Psi_{\text {val }}(\boldsymbol{r}) .
\end{aligned}
$$

Here we have defined the bilocal kernel

$$
\begin{aligned}
\tilde{\mathcal{K}}\left(r, r^{\prime}\right)= & \frac{4 \pi}{3} \frac{m_{\omega}^{2} f_{\pi}^{2}}{m^{2}} \frac{\delta\left(r-r^{\prime}\right)}{r^{2}} \\
& +N_{C} \eta_{\mathrm{val}} \sum_{\nu \neq \text { val }} \int d \Omega \int d \Omega^{\prime} \Psi_{\nu}^{\dagger}\left(\boldsymbol{r}^{\prime}\right) \Psi_{\mathrm{val}}\left(\boldsymbol{r}^{\prime}\right) \frac{1}{\epsilon_{\nu}^{0}-\epsilon_{\mathrm{val}}^{0}} \Psi_{\mathrm{val}}^{\dagger}(\boldsymbol{r}) \Psi_{\nu}(\boldsymbol{r}) \\
& +\frac{N_{C}}{2} \sum_{\nu \mu} \int d \Omega \int d \Omega^{\prime} \Psi_{\nu}^{\dagger}\left(\boldsymbol{r}^{\prime}\right) \Psi_{\mu}\left(\boldsymbol{r}^{\prime}\right) \tilde{f}\left(\epsilon_{\nu}^{0}, \epsilon_{\mu}^{0} ; \Lambda\right) \Psi_{\mu}^{\dagger}(\boldsymbol{r}) \Psi_{\nu}(\boldsymbol{r}) .
\end{aligned}
$$

Since $\Psi_{\nu}(\boldsymbol{r})=\langle\boldsymbol{r} \mid \nu\rangle$ denote the coordinate representations of the eigenstates $|\nu\rangle$ of $h_{\Theta}$ this kernel functionally depends on the chiral angle $\Theta$. Obviously $\tilde{\mathcal{K}}\left(r, r^{\prime}\right)$ is symmetric in its arguments. The RHS of the equation of motion (5.6) is, of course, nothing but the quark baryon density in the regularized form. Upon integration of this equation one easily verifies the normalization condition (4.7). Here the important ingredient is again the fact that the regulator functions $\tilde{f}$ has vanishing diagonal elements. By the virial theorem one can then eliminate the linear term in the Minkowski energy functional for profiles which satisfy (5.6)

$$
E_{2}^{\mathrm{Mink}}\left[\Theta, \omega_{0}\right]=E_{0}[\Theta]+\frac{1}{2} \int d r r^{2} \int d r^{\prime} r^{\prime 2} \tilde{\mathcal{K}}\left(r, r^{\prime}\right) \omega_{0}(r) \omega_{0}\left(r^{\prime}\right)
$$

\footnotetext{
$\ddagger$ This is due to the fact that the perturbation of the wave-function is orthogonal to the unperturbed state in Rayley-Schrödinger perturbation theory.

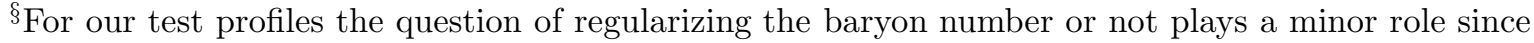
numerically the regularized baryon number comes out to be 0.96 .
} 
Only in case that all eigenvalues of this kernel are positive the Minkowski energy is positive definite. For the numerical treatment we have discretized the radial coordinate leaving this kernel as a matrix $\tilde{\mathcal{K}}\left(r, r^{\prime}\right) \rightarrow \tilde{\mathcal{K}}_{i j}$. For various test profiles $\Theta$ we have found that this matrix possesses negative eigenvalues. This can easily be understood by considering $\tilde{f}$ in eqn (5.5). The dominant contribution comes from states with $\epsilon_{\nu} \approx \epsilon_{\mu}$. Assuming $\epsilon_{\nu}>\epsilon_{\mu}$ (without loss of generality since $\tilde{f}$ is symmetric) we see that the denominator is positive. On the other hand the numerator is negative because the complementary error function decreases monotonically with the argument. Thus $\tilde{f}$ may assume large negative values. Stated differently, the fact that the Minkowski energy functional is not positive definite for profiles satisfying the stationary condition (5.6) is a consequence of the discontinuity of $\tilde{f}\left(\epsilon_{\nu}, \epsilon_{\mu} ; \Lambda\right)$ as $\epsilon_{\mu} \rightarrow \epsilon_{\nu}$.

We have thus seen that counting powers of the $\omega$-field in the eigenvalues of the Hamiltonian $h_{\Theta}+i \omega_{4}$ leads to an ill-defined expression for the real part of the fermion determinant in the proper-time regularization (1.9). We also conjecture that the nonpositiveness of the energy functional based on this power counting represents the reason why the authors of ref. 21] were unable to find self-consistent solutions for physically relevant parameters.

To resolve this problem let us next examine $\mathcal{A}_{R}$ in more detail. In order to expand $\mathcal{A}_{R}$ in terms of $\omega_{4}$ one starts off at the module

$$
\not D_{E} \not D_{E}^{\dagger}=-\partial_{\tau}^{2}+h_{\Theta}^{2}+2 i \omega_{4} \partial_{\tau}+i\left[h_{\Theta}, \omega_{4}\right]+\omega_{4}^{2}
$$

Performing subsequently an expansion of

$$
\begin{aligned}
\mathcal{A}_{R} & =\frac{1}{2} \operatorname{Tr} \log \left(-\partial_{\tau}^{2}+h_{\Theta}^{2}+2 i \omega_{4} \partial_{\tau}+i\left[h_{\Theta}, \omega_{4}\right]+\omega_{4}^{2}\right) \\
& \rightarrow-\frac{1}{2} \operatorname{Tr} \int_{1 / \Lambda^{2}}^{\infty} \frac{d s}{s} \exp \left(-\partial_{\tau}^{2}+h_{\Theta}^{2}+2 i \omega_{4} \partial_{\tau}+i\left[h_{\Theta}, \omega_{4}\right]+\omega_{4}^{2}\right)
\end{aligned}
$$

corresponds to imposing the proper-time regularization scheme at the operator level. As has already been explained in section 3 the regularized form (3.15) follows from an intermediate definition of the proper-time regularization and involves the assumption of simultaneous diagonalizability of $h$ and $h^{\dagger}$. This is exactly the point where $\omega_{4}-$ power counting of the eigenvalues fails to provide the correct result for the expansion of $\mathcal{A}_{R}$. We therefore expect different results for the action functional when adopting (5.11) as starting point for expanding in $\omega_{4}$ rather than expanding the energy eigenvalues. Note that the expression (5.9) is still exact in $\omega_{4}$, i.e. no expansion has been performed up to here. The vacuum contribution to the real part of the fermion determinant is then expanded up to quadratic order in $\omega_{4}$ by methods which have previously been worked out in the context of quantizing the chiral soliton [27] and has subsequently been extended to study fluctuations off the chiral soliton [29]. We refer the interested reader to the appendix where the calculation is performed. The net result of this calculation is that the regulator function $\tilde{f}$ defined in eqn (5.5) has to substituted by

$$
f\left(\epsilon_{\mu}, \epsilon_{\nu} ; \Lambda\right)=\frac{1}{2} \frac{\operatorname{sgn}\left(\epsilon_{\mu}\right) \operatorname{erfc}\left(\left|\frac{\epsilon_{\mu}}{\Lambda}\right|\right)-\operatorname{sgn}\left(\epsilon_{\nu}\right) \operatorname{erfc}\left(\left|\frac{\epsilon_{\nu}}{\Lambda}\right|\right)}{\epsilon_{\mu}-\epsilon_{\nu}}-\frac{\Lambda}{\sqrt{\pi}} \frac{e^{-\left(\epsilon_{\mu} / \Lambda\right)^{2}}-e^{-\left(\epsilon_{\nu} / \Lambda\right)^{2}}}{\epsilon_{\mu}^{2}-\epsilon_{\nu}^{2}} .
$$

The corresponding replacements have also to be performed in the equations (5.4) and (5.7). The latter replacement defines the bilocal kernel $\mathcal{K}$ which then enters the new 
equation of motion and energy functionals obtained by the corresponding substitutions in equations (5.6) and (5.8), respectively. Apparently these two regulator functions $(\tilde{f}$ and $f$ ) only agree in the infinite cut-off limit, $\Lambda \rightarrow \infty$. This is not astonishing because in this limit the introduction of the Hermitian conjugate of the Dirac operator is not mandatory in order to (formally) evaluate the fermion determinant. It is also noteworthy that the regulator function $f(5.12)$ is identical to the one which appears in the moment of inertia when the semi-classical cranking approach is applied to quantize the chiral soliton [27. This is intuitively clear because for that problem the perturbation (proportional to the isospin generators) in the Dirac Hamiltonian is also static and anti-Hermitian. In that context it has been shown that the limit

$$
\lim _{\epsilon_{\mu} \rightarrow \epsilon_{\nu}} f\left(\epsilon_{\mu}, \epsilon_{\nu} ; \Lambda\right)=0
$$

is assumed continuously (see chapter 5 in ref. 227). Numerically we have also verified that the associated kernel $\mathcal{K}$ possesses positive eigenvalues only. Thus the corresponding energy functional is positive definite for $\omega$ profiles which statisfy the modified equation of motion $(\mathcal{K}$ inserted in eqn (5.6)). Due to the limit (5.13) it is clear that the Minkowski energy functional with $\tilde{f}$ replaced by $f$ satisfies the consistency conditions derived in section 4. For our standard test profile we find that the second order in $\omega_{0}$ contributes about $-129 \mathrm{MeV}$ to the Minkowski energy. This is significantly smaller (in magnitude) than for the regularization function $\tilde{f}$ (see table 5.2). Thus the numerically results put further doubts on the validity of counting $\omega_{4}$ powers in the energy eigenvalues. On the other hand, employing the correct regulator function $f$ not only satisfies our consistency conditions but also provides a quickly converging series. The zeroth and first order contributions may be read off from table 5.2. Thus we have, for the first time, available a well suited Minkowski energy functional for the NJL model which includes the $\omega$ meson. For completeness and later reference we list it here again although the (formal) deviation from (5.4) is only minor

$$
\begin{array}{r}
E^{\operatorname{Mink}}\left[\Theta, \omega_{0}\right]=E_{0}[\Theta]+N_{C} \eta_{\text {val }}\left\langle\operatorname{val}\left|\omega_{0}\right| \operatorname{val}\right\rangle-\frac{N_{C}}{2} \sum_{\nu} \operatorname{sgn}\left(\epsilon_{\nu}^{0}\right) \operatorname{erfc}\left(\left|\frac{\epsilon_{\nu}^{0}}{\Lambda}\right|\right)\left\langle\nu\left|\omega_{0}\right| \nu\right\rangle \quad(5.14) \\
-N_{C} \eta_{\text {val }} \sum_{\nu \neq \text { val }} \frac{\mid\left.\left\langle\nu\left|\omega_{0}\right| \text { val }\right\rangle\right|^{2}}{\epsilon_{\nu}^{0}-\epsilon_{\text {val }}^{0}}-\frac{N_{C}}{2} \sum_{\nu \mu} f\left(\epsilon_{\nu}^{0}, \epsilon_{\mu}^{0} ; \Lambda\right)\left|\left\langle\nu\left|\omega_{0}\right| \mu\right\rangle\right|^{2}-\frac{4 \pi}{3} \frac{m_{\omega}^{2} f_{\pi}^{2}}{m^{2}} \int d r r^{2} \omega_{0}^{2} .
\end{array}
$$

For $\omega$ profile which statisfy the associated stationary condition this can be decomposed as

$$
E_{2}^{\mathrm{Mink}}\left[\Theta, \omega_{0}\right]=E_{0}[\Theta]+E_{\omega}[\Theta, \omega]
$$

where

$$
E_{\omega}[\Theta, \omega]=\frac{1}{2} \int d r r^{2} \int d r^{\prime} r^{\prime 2} \mathcal{K}\left(r, r^{\prime}\right) \omega_{0}(r) \omega_{0}\left(r^{\prime}\right)
$$

denotes the contribution due to the $\omega$ field.

In the next step one has to construct the self-consistent solution which extremizes the functional (5.14). Unfortunately this is not an easy task. The reason is that the kernel $\mathcal{K}$ functionally depends on the chiral angle $\Theta$. Thus the stationary condition for $\Theta$ requires the functional derivative of the wave-functions $\Psi_{\nu}(\boldsymbol{r})$ with respect to $\Theta$. Such a calculation goes beyond the scope of the present paper and we will pursue a 
Table 5.3: Parameters describing the energy minimum as well the corresponding total energy in Minkowski space, see eqn (5.14). Also shown is the contribution of the $\omega$ field (5.16).

\begin{tabular}{|c|ccc|ccc|}
\hline$m(\mathrm{MeV})$ & $\lambda^{\text {min }}$ & $E^{\text {Mink }}(\mathrm{MeV})$ & $E_{\omega}(\mathrm{MeV})$ & $R_{m}^{\min }(\mathrm{fm})$ & $E^{\mathrm{Mink}}(\mathrm{MeV})$ & $E_{\omega}(\mathrm{MeV})$ \\
\hline 400 & 0.852 & 1586 & 321 & 0.713 & 1590 & 317 \\
450 & 0.776 & 1685 & 380 & 0.775 & 1685 & 378 \\
500 & 0.729 & 1769 & 428 & 0.830 & 1768 & 419 \\
\hline
\end{tabular}

different approach to find the energy minimum. First, we introduce parameters $\{\chi\}$ to describe the shape of the profile function $\Theta(r)$ and evaluate the corresponding eigenvalues and -energies of $h_{\Theta}$. These are then functions of the parameters $\{\chi\}$. In a second step this allows to construct the kernel $\mathcal{K}$ as a function of these parameters as well. Via the stationary condition for $\omega_{0}$, eqn (5.6) with $\tilde{f}$ replaced by $f$, also $\omega_{0}$ becomes a function of these parameters. As already mentioned in the introduction this stationary condition commonly represents a constraint rather than an equation of motion. This fact furthermore justifies our procedure to parametrize the chiral angle while solving exactly for the $\omega$ profile. Technically this stationary condition is an inhomogeneous integral equation which is solved by inverting the discretized bilocal kernel $\mathcal{K}_{i j}$. Finally the parameters are tuned such that $E^{\text {Mink }}$ acquires a minimum. Since the main effect of the $\omega$ meson on the chiral angle is of repulsive character it is intuitive to introduce a parameter which allows to vary the spatial extension of $\Theta(r)$. In a first calculation we therefore introduce the breathing mode parameter $\chi=\lambda$ via

$$
\Theta_{\lambda}(r)=\Theta_{\text {s.c. }}(\lambda r)
$$

where $\Theta_{\text {s.c. }}(r)$ denotes the self-consistent soliton solution to the problem without the $\omega$ meson. The resulting energy is displayed on left of figure 5.1 as a function of $\lambda$. Secondly we have adopted the description [31]

$$
\Theta_{R_{m}}(r)=-\pi \begin{cases}1-\frac{r}{2 R_{r}}, & r \leq R_{m} \\ \left(1-\frac{R_{m}}{2 R_{r}}\right)\left(\frac{R_{m}}{r}\right)^{2} \frac{1+m_{\pi} r}{1+m_{\pi} R_{m}} \exp \left(m_{\pi}\left(R_{m}-r\right)\right), & r \geq R_{m}\end{cases}
$$

for the chiral angle. Since $R_{r}$ is chosen such that the derivative of $\Theta$ is continuous the chiral angle only depends on $R_{m}$ parametrically. The results for the energy corresponding from the parametrization (5.18) may also be found in figure 5.1. Actually these two parametrizations yield almost identical results. For a constituent quark mass $m<400 \mathrm{MeV}$ no local minimum is obtained and the trivial minimum $(E=3 m)$ corresponding to three free quarks is assumed. In the latter case the chiral angle gets very narrow while the $\omega$-field tends to zero except within a vicinity of the origin such that $\left|\int d^{3} r \omega\right|>0$ and $\left|\int d^{3} r \omega^{2}\right| \rightarrow 0$. As the constituent quark mass is increased the local minimum gets more pronounced. In table 5.3 we list the parameters describing the minimum as well as the resulting total energy. There $\lambda^{\text {min }}$ and $R_{m}^{\min }$ denote the parameters associated with the energy minimum. As already mentioned after eqn (4.7) the increase of the static energy due to the inclusion of the $\omega$ meson is no surprise although the number of degrees of freedom has been enlarged. The fact that at the minimum we always find $\lambda<1$ corroborates the repulsive character of the $\omega$ meson. Now we are finally able to mention that the test profiles, which we have been using all the time, correspond to the 
parameterization (5.17) for $m=400 \mathrm{MeV}$ and $\lambda=0.86$, i.e. a configuration which seems to be very close to the stationary solution. Table 5.3 also contains the contribution of the $\omega$ meson to the energy as defined in eqn (5.16). The fact that this quantity is more sensible to the chosen parametrization than the total energy indicates that a parameter ansatz for the soliton profiles is suitable to compute the minimal energy but some refinements may be necessary when studying other quantities, e.g. radii, magnetic moments, etc. . In figure 5.2 the profile functions which minimize $E^{\mathrm{Mink}}$ in the parametrization (5.17) are plotted. In this figure the quark baryon density, $b \propto R H S$ of eqn (5.6), is scaled such that the spatial integrals over $\omega / m$ and $b$ coincide. Obviously the $\omega$ profile is enhanced over $b$ at large distances leading to a larger isoscalar radius in agreement with assertion (1.3). The fact that the presence of the $\omega$ meson causes the soliton configuration to swell has also the consequences that the axial charge becomes larger and that the valence quark gets bound more strongly. These results were also obtained in the approach based on the linear approximation (4.12) [24]. We have furthermore considered fluctuations around the profile $\Theta_{R_{m}^{\min }}(r)$ in order to obtain an improved solution. The best we were able to achieve was a further decrease of the total energy by about $5 \mathrm{MeV}$, i.e. the parametrizations (5.17) and (5.18) allow for a good approximation to exact minimum.

We should also note that we have performed the analogous calculations using the regulator function $\tilde{f}$. In that case no local minimum was obtained. This provides a further indication why the approach of ref. 21] did not lead to self-consistent solutions.

Here we will not further discuss the results for various observables in this approach but rather make some remarks on the case when the isovector mesons $\rho$ and $a_{1}$ are included. The previous formulas apply as well, however, the Hermitian part of the Hamiltonian has to be supplemented [19,

$$
\begin{aligned}
h_{\Theta}= & \boldsymbol{\alpha} \cdot \boldsymbol{p}+m \beta\left(\cos \Theta(r)+i \gamma_{5} \boldsymbol{\tau} \cdot \hat{\boldsymbol{r}} \sin \Theta(r)\right) \\
& +\frac{1}{2}(\boldsymbol{\alpha} \times \hat{\boldsymbol{r}}) \cdot \boldsymbol{\tau} G(r)+\frac{1}{2}(\boldsymbol{\sigma} \cdot \hat{\boldsymbol{r}})(\boldsymbol{\tau} \cdot \hat{\boldsymbol{r}}) F(r)+\frac{1}{2}(\boldsymbol{\sigma} \cdot \boldsymbol{\tau}) H(r) .
\end{aligned}
$$

The radial functions $G(r), F(r)$ and $H(r)$ denote the vector $\left(V_{\mu}=\boldsymbol{V}_{\mu} \cdot \boldsymbol{\tau} / 2\right)$ and axial vector $\left(A_{\mu}=\boldsymbol{A}_{\mu} \cdot \boldsymbol{\tau} / 2\right)$ meson profiles

$$
V_{i}^{a}=\epsilon_{a k i} \hat{r}_{k} G(r) \quad A_{i}^{a}=\delta_{i a} H(r)+\hat{r}_{i} \hat{r}_{a} F(r) \quad(i, a=1,2,3) .
$$

As the number of fields involved increases the parametrization of the profile functions entering the Hermitian part of the Hamiltonian get more ambiguous. Motivated by the breathing mode description one might, in addition to (5.17), assume the ansätze

$$
\Phi_{\lambda}(r)=\lambda^{n} \Phi_{\text {s.c. }}(\lambda r) \quad \text { for } \quad \Phi=F, G, H .
$$

The choice $n=0$ would be analogous to (5.17) while $n=1$ is suggested by the asymptotic form of the (axial) vector meson fields in terms of the chiral angle.

Two important changes in comparison to the previous studies have to be made in order to compute the total energy when the $\rho$ and $a_{1}$ fields are included. First the mixing between the pions and the axial vector meson field leads to a different relation between the cut-off $\Lambda$ and the pion decay constant $f_{\pi}$ [15]

$$
f_{\pi}^{2}=\frac{N_{C} m^{2}}{4 \pi^{2}} \Gamma\left(0,\left(\frac{m}{\Lambda}\right)^{2}\right) \frac{m_{\rho}^{2}}{m_{\rho}^{2}+6 m^{2}} .
$$


Table 5.4: The position $\left(\lambda^{\mathrm{min}}\right)$ and the value $\left(E^{\mathrm{Mink}}\right)$ of the local minimum of the energy when the vector mesons are included. Also shown is the contribution of the $\omega$ field (5.16). The constituent quark mass $m=350 \mathrm{MeV}$ is assumed.

\begin{tabular}{|cc|cc|}
\hline$n$ & $\lambda^{\text {min }}$ & $E^{\text {Mink }}(\mathrm{MeV})$ & $E_{\omega}(\mathrm{MeV})$ \\
\hline 0.00 & 0.850 & 1662 & 553 \\
0.25 & 0.800 & 1622 & 490 \\
0.50 & 0.745 & 1565 & 418 \\
0.75 & 0.680 & 1501 & 340 \\
1.00 & 0.630 & 1433 & 285 \\
1.25 & 0.600 & 1387 & 253 \\
1.50 & 0.603 & 1376 & 249 \\
1.75 & 0.625 & 1385 & 259 \\
2.00 & 0.655 & 1403 & 273 \\
\hline
\end{tabular}

This increases the value for $\Lambda$ once a constituent quark mass $m$ is given. Secondly the vector meson mass term becomes [24]

$$
\frac{m_{\rho}^{2}}{2 \pi} \Gamma\left(0,\left(\frac{m}{\Lambda}\right)^{2}\right) \int d r r^{2}\left[G^{2}(r)+\frac{1}{2} F^{2}(r)+F(r) H(r)+\frac{3}{2} H^{2}(r)-2 \omega_{0}^{2}(r)\right] .
$$

We are now enabled to search for a local minimum using the parametrization (5.21). In table 5.4 we present values $\lambda^{\text {min }}$ of the breathing mode variable which lead to a local minima as well as the corresponding energies in the interval $0 \leq n \leq 2$. Here we use the constituent quark mass $m=350 \mathrm{MeV}$ and assume the imaginary part in the unregularized from $₫$. As a reminder we should mention that without the $\omega$ meson this soliton energy is obtained to be $1010 \mathrm{MeV}$ [19]. We observe that a local minimal in the energy surface is obtained for $n \approx 1.5$ and $\lambda \approx 0.58$. Upon further increase of $n$ the energy increases rather slowly. In the same way the value of the scaling parameter corresponding to the minimal energy, $\lambda^{\mathrm{min}}$, gets larger again. The minimum of the total energy is also characterized by the smallest contribution from the $\omega$ meson. It should be noted that the minimal energy $(1376 \mathrm{MeV})$ is still $326 \mathrm{MeV}$ above the energy of the trivial $B=1$ configuration where three (almost) free valence quark orbits are occupied. Again we observe the repulsive character of the $\omega$ meson reflected by the fact that $\lambda^{\text {min }}<1$. In contrast to the case with only the pseudoscalar fields this leads to a somewhat smaller binding of the valence quarks. We find $\epsilon_{\text {val }}^{0} / m=-0.29$ to be compared with -0.38 in the model without the $\omega$ [19. This reduction seems to be due the diminishing profile functions of the (axial) vector mesons. A similar behavior has been obtained when extremizing the energy functional involving the definition (4.13) in the case that the imaginary has not been regularized [24. As this prescription is also adopted here we recognize at least qualitative similarities. The value for the axial charge of the nucleon as obtained from the integral [24]

$$
g_{A}=-\frac{2 \pi}{3 g_{2}} \int d r r^{2}\left[H(r)+\frac{1}{3} F(r)\right]
$$

increases from 0.27 to 0.48 by the inclusion of the $\omega$ meson. This is, of course, a correction into the right direction but nevertheless only about $1 / 3$ of the empirical value extracted

"When $\rho$ and $a_{1}$ fields are included the regularized baryon number deviates strongly from unity [24] in contrast to the model with pseudoscalars only. 
from neutron $\beta$-decay. It has been argued that $1 / N_{C}$ corrections may further improve on the result for $g_{a}$ [32]; these arguments have, however, to be taken with some care in respect to the symmetries of the model [33].

As the main subject of the present paper is the investigation of the $\omega$ meson in the context of the energy functional we will not go into detail on the nucleon properties. Especially because we only have a parametrical description of the stationary field configuration rather than a self-consistent solution. The nucleon properties are most likely more sensitive to the shape of the meson profiles and the quark wave-functions than the soliton energy. Nevertheless the construction of the self-consistent solution would be an interesting path to pursue in particular in order to gain information about the nucleon properties. In this context we also wish to remark that the $\omega$ profile associated with the minimal energy configuration $\left(n=1.5, \lambda^{\min }=0.598\right)$ does not turn out to be a smooth radial function but rather possesses small fluctuations off a monotonously decreasing function. We expect an exact solution to improve in this respect.

\section{Summary and Conclusions}

In this paper we have performed a thorough study of the analytical properties of a fermion determinant for the case that the $\omega$-field is present in the static hedgehog configuration. This has recently been a matter of dispute in the context of the soliton in the NJL model with (axial) vector meson degrees of freedom. The fact that this model requires regularization makes mandatory the continuation forth and back from Minkowski to Euclidean space. This continuation represents an element of the transformations we have been considering in the complex $z$-plane defined by the Dirac Hamiltonian (2.4). As originally the fermion determinant is expressed in terms of the eigenvalues of this nonHermitian operator we first have investigated the behavior of the associated eigenvalues in the $z$-plane. Thereby we have confirmed an earlier result [26] that for physically motivated field configurations these eigenvalues are smooth functions along the path connecting Euclidean and Minkowski spaces. Furthermore we have demonstrated that the Laurent series of such an eigenvalue or -function indeed reduces to a Taylor series. In this way the analyticity of eigenvalues and -functions is verified. Such an analytic behavior is not a priori clear because the eigenvalues are roots of a polynomial of large degree. We have, however, encountered a different kind of non-analyticity which is caused by a level crossing. Such crossing may appear if the $\omega$-field is sufficiently pronounced. Thus, in order to attach physical significance to a given field configuration one has to check that no such level crossing takes place along the path from Euclidean to Minkowski spaces.

In the next step we have then examined the behavior of the full fermion determinant in the complex $z$-plane. Formally analytical one-particle eigenvalues yield an analytical determinant in the unregularized form. However, regularization is unavoidable and introduces (at least in the proper-time scheme) the Hermitian conjugate of the Euclidean

Dirac operator $\not_{E}^{\dagger}$. As a consequence the real and imaginary parts of the one-particle eigenvalues are treated differently when summing up for the fermion determinant. This represents the main reason for the fermion determinant to be non-analytical in $z$. As a matter of fact the corresponding Laurent series (3.25, 3.26) has vanishing radius of convergence. We therefore conclude that the analytical continuation of the Euclidean action functional to Minkowski space does not exist. This makes a derivation of the Minkowski energy functional by means of analytic continuation impossible.

In order to nevertheless obtain a sensible definition of a Minkowski energy functional we have then formulated two consistency conditions to be satisfied by this functional. 
The first one says that the energy changes by $N_{C} B \omega_{c}$ under a constant shift $\left(\omega_{c}\right)$ of the $\omega$-field as a consequence of global gauge invariance ( $B$ is the baryon number.). Secondly, the current field identity for the baryon current imposes a normalization on the $\omega$-field (4.7). We have then examined the three treatments known in the literature with respect to these conditions. In this context only two approaches (by Schüren et al. 221, 22] and the one by the present authors [23, 24]) were shown to be consistent. The reason is that these two approaches are motivated by the Euclidean energy functional in contrast to the treatment by Watabe and Toki [20].

In the last section we have then investigated the expansion of the fermion determinant in powers of the $\omega$-field. In particular we have examined the role of $\not D_{E}^{\dagger}$. For this purpose we have considered the $\omega$-field as a perturbation to the Euclidean Dirac Hamiltonian and applied standard perturbation techniques. We have then demonstrated that at second order the bilocal kernel $\tilde{\mathcal{K}}$ in eqn (5.7) is not positive definite putting some doubts on the validity on an approach which is based on counting $\omega_{4}$ powers in the eigenvalues $\epsilon_{\nu}$. The reason is that the corresponding regularization function $\tilde{f}\left(\epsilon_{\nu}, \epsilon_{\mu} ; \Lambda\right)$ does not smoothly assume the limit $\tilde{f} \rightarrow 0$ as $\epsilon_{\mu} \rightarrow \epsilon_{\nu}$. A vanishing regularization function for identical arguments is required in order to satisfy the consistency conditions derived in section 4 . As a matter of fact the functional trace of the operator $D_{E} \not D_{E}^{\dagger}$ rather than the eigenvalues of the Dirac Hamiltonian is required in order to evaluate the fermion determinant in the proper-time regularization scheme. Stated otherwise, the proper-time prescription has to be imposed at the operator level. This point of view has already been adopted previously when performing the semi-classical quantization for two 27] and three flavors [37. We have then observed that imposing the proper-time prescription at the operator level leads to a different expansion in terms of the $\omega$-field for the action functional. From a mathematical point of view there are two ways for understanding the different results. First, in order to obtain the regularized form of the energy functional (3.15) from the regularized action (1.9) one has to make the inexact assumption that the Euclidean Dirac Hamiltonian and its complex conjugate can be diagonalized simultaneously. It is then also clear that both expansion schemes yield identical results when the perturbation of the Dirac Hamiltonian is static and Hermitian. We have verified this for an expansion up to second order. The second explanation relies on the fact that due to regularization the Hilbert space gets restricted and thus the rules for handling logarithms are no longer valid. Adopting this explanation it becomes also clear that in the limit $\Lambda \rightarrow \infty$ (no regularization) the two expansion schemes are again identical. In regard of the fact that the derivation of the functional (3.11) involves a non-converging quantity, the results for the semi-classical quantization [27, 37], the smoothness of the regulator function $f$ (5.12) and the existence of a local minimum in the energy surface (5.14) we consider the imposition of the proper-time regularization prescription at the operator level as the correct one. This conclusion finally prohibits an expansion of the eigenvalues $\epsilon_{\nu}$ in order to obtain the Minkowski energy functional in the presence of the $\omega$ meson. We have also seen that a series based on the expansion of $\not_{E} \not D_{E}^{\dagger}$ converges rather quickly (at least at second order).

This conclusion has enforced us to investigate the corresponding energy functional (5.14) in more detail. Employing a parametrical description of the chiral angle we have constructed the local minimum to this functional. Local minima exist for constituent

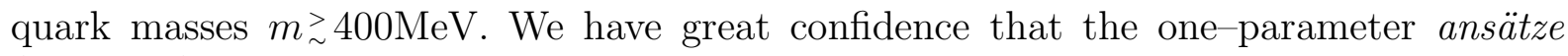
(5.17,5.18) describe the exact solution quite well. For these solutions we have confirmed 
the repulsive character of the $\omega$-field. When other vector meson fields entering the Hermitian part of the Dirac Hamiltonian are included as well, a parametrical description of the Dirac Hamiltonian is more ambiguous. Nevertheless we have demonstrated that for the constituent quark mass $m=350 \mathrm{MeV}$ a solution exists. In particular, the result that the valence quark level joins the Dirac vacuum, which was previously obtained in calculations ignoring the $\omega$ meson [19], is affirmed. This is again mainly due to the repulsive character of the isoscalar $\omega$.

Let us finally add a note on similar soliton models which include the $\omega$ meson. In extended Skyrme models [11, 30, 34, 35] or the chiral quark model 36] the $\omega$ meson appears only in terms of explicit polynomials which renders the energy functional analytic in the complex $z$-plane. More importantly these models do actually not require regularization abandoning the need for continuation forth and back from Minkowski to Euclidean spaces.

In order to point out an interesting task to pursue in future we should mention that we have not yet solved the complete set of stationary conditions in the presence of all meson fields. In the present work we have only made plausible that such a solution exists. However, a calculation aimed at the extremization of the functional (5.14) with all meson profiles included, which are compatible with the grand spin and parity symmetries of the hedgehog ansatz, is highly desirable because we have (for the first time) available a reasonable approximation (5.14) to the energy functional of the NJL soliton model including all relevant meson fields. This should represent a well suited model for the description of static nucleon properties.

\section{Acknowledgement}

One of us (HW) gratefully acknowledges a fruitful conversation with C. Schüren, E. Ruiz Arriola and K. Goeke at the ETC* workshop in Trento on The Quark Structure of Baryons, October 1993.

\section{Appendix: Regularization for Quadratic Terms}

In this expansion we wish to describe the expansion of the vacuum part of the Euclidean energy functional in terms of the isoscalar vector $\omega$-meson. The starting point is represented by the Euclidean Dirac operator

$$
i \beta \not D_{E}=-\partial_{\tau}-h=-\partial_{\tau}-h_{\Theta}-i \omega_{4}
$$

with the Dirac Hamiltonian being decomposed into Hermitian $\left(h_{\Theta}\right)$ and anti-Hermitian $\left(i \omega_{4}\right)$ pieces, see also eqn (2.1). In order to extract the vacuum part we will only consider the limit of large Euclidean times $T \rightarrow \infty$. We actually need to expand

$$
\mathcal{A}_{E}=\operatorname{Tr} \log \left(-\partial_{\tau}-h_{\Theta}-i \omega_{4}\right)
$$

in powers of the $\omega_{4}$ field: $\mathcal{A}_{E}=\mathcal{A}_{E}^{0}+\mathcal{A}_{E}^{1}+\mathcal{A}_{E}^{2}+\ldots$. Here the superscripts label the order in which the $\omega$ field appears. The term linear in $\omega_{4}$ is straightforwardly obtained to become purely imaginary

$$
\mathcal{A}_{E}^{1}=-i \operatorname{Tr}\left[\left(\partial_{\tau}+h_{\Theta}\right)^{-1} \omega_{4}\right] .
$$


In the limit of large Euclidean times $(T \rightarrow \infty)$ the temporal part of the trace is replaced by a spectral integral while the trace over the remaining degrees of freedom is carried out using the eigenstates of $h_{\Theta}(c f$. section 3$)$. We would like to remark that the corresponding eigenvalues $\epsilon_{\nu}^{0}$ are real. Then one easily finds

$$
\mathcal{A}_{E}^{1}=-i N_{C} \sum_{\nu} \mathcal{P} \int \frac{d v}{2 \pi}\left(i v+\epsilon_{\nu}^{0}\right)^{-1}\left\langle\nu\left|\omega_{4}\right| \nu\right\rangle=-i N_{C} \sum_{\nu} \int_{-\infty}^{\infty} \frac{d v}{2 \pi} \frac{\epsilon_{\nu}}{v^{2}+\left(\epsilon_{\nu}^{0}\right)^{2}}\left\langle\nu\left|\omega_{4}\right| \nu\right\rangle .(
$$

Again one may introduce a finite proper-time cut-off $\Lambda$ by expressing the denominator in eqn (A.4) in terms of a parameter integral

$$
\mathcal{A}_{E}^{1}=-i N_{C} T \sum_{\nu} \epsilon_{\nu}^{0} \int_{-\infty}^{\infty} \frac{d v}{2 \pi} \int_{1 / \Lambda^{2}}^{\infty} d s \exp \left[-s\left(v^{2}+\left(\epsilon_{\nu}^{0}\right)^{2}\right)\right]\left\langle\nu\left|\omega_{4}\right| \nu\right\rangle .
$$

The spectral integral may now be performed which results in

$$
\mathcal{A}_{E}^{1}=\frac{-i}{2} N_{C} T \sum_{\nu} \operatorname{sgn}\left(\epsilon_{\nu}^{0}\right) \operatorname{erfc}\left(\left|\frac{\epsilon_{\nu}^{0}}{\Lambda}\right|\right)\left\langle\nu\left|\omega_{4}\right| \nu\right\rangle .
$$

At this point is important to remark that this result can also be gained by the application of standard perturbation techniques to the expression for the Euclidean energy functional obtained in section 3. In a first step one expands the functional (3.24) for $z=i$ and $z^{*}=-i$ in terms of the one-particle eigenenergies. Secondly, standard perturbation theory is employed to make the $\omega_{4}$ dependence explicit as described in eqns (5.1) and (5.2). Carrying out this procedure up to linear order in $\omega_{4}$ exactly reproduces eqn (A.6). The term of quadratic order $\epsilon_{\nu}^{2}$ will be considered later.

Next we turn to the evaluation of $\mathcal{A}_{E}^{2}$. As the terms of even powers in $\omega_{4}$ contribute to the real part of the fermion determinant we may start the definition of the proper-time regularization (1.9)

$$
\mathcal{A}_{R}=-\frac{1}{2} \operatorname{Tr} \int_{1 / \Lambda^{2}}^{\infty} \frac{d s}{s} \exp \left(-s D_{E} D_{E}^{\dagger}\right)
$$

In the presence of the $\omega$ meson the operator $D_{E} \not_{E}^{\dagger}$ is presented in eqn (5.9). We then apply techniques which have previously been developed for the description of meson fluctuation off the soliton 29 to this expression. As the $\omega$ field is assumed to be static the expansion is also similar to the $1 / N_{C}$ expansion performed to extract the moment of inertia in the cranking approach [27]. The non-vanishing contributions at quadratic order in $\omega_{4}$ are found to be

$$
\begin{aligned}
\mathcal{A}_{R}^{2}= & \frac{1}{2} \operatorname{Tr} \int_{1 / \Lambda^{2}}^{\infty} d s \exp \left[-s\left(h_{\Theta}^{2}-\partial_{\tau}^{2}\right)\right] \omega_{4}^{2} \\
& -\frac{1}{2} \operatorname{Tr} \int_{1 / \Lambda^{2}}^{\infty} d s s \int_{0}^{1} d x \int_{0}^{1-x} d y \exp \left[-s x\left(h_{\Theta}^{2}-\partial_{\tau}^{2}\right)\right]\left\{2 i \omega_{4} \partial_{\tau}+i\left[h_{\Theta}, \omega_{4}\right]\right\} \\
& \times \exp \left[-s(1-x-y)\left(h_{\Theta}^{2}-\partial_{\tau}^{2}\right)\right]\left\{2 i \omega_{4} \partial_{\tau}+i\left[h_{\Theta}, \omega_{4}\right]\right\} \exp \left[-s y\left(h_{\Theta}^{2}-\partial_{\tau}^{2}\right)\right] .
\end{aligned}
$$

One of the Feynman parameter integrals can be carried out trivially due to the cyclic properties of the trace. Furthermore the temporal part of the trace turns into a spectral 
integral for $T \rightarrow \infty$.

$$
\begin{aligned}
\mathcal{A}_{R}^{2}= & \frac{1}{2} T \operatorname{tr} \int_{1 / \Lambda^{2}}^{\infty} d s \int_{-\infty}^{\infty} \frac{d v}{2 \pi} \exp \left[-s\left(h_{\Theta}^{2}+v^{2}\right)\right] \omega_{4}^{2} \\
& -\frac{1}{4} T \operatorname{tr} \int_{1 / \Lambda^{2}}^{\infty} d s s \int_{-\infty}^{\infty} \frac{d v}{2 \pi} \int_{0}^{1} d \alpha \exp \left[-s \alpha\left(h_{\Theta}^{2}+v^{2}\right)\right]\left\{2 \omega_{4} v+i\left[h_{\Theta}, \omega_{4}\right]\right\} \\
& \times \exp \left[-s(1-\alpha)\left(h_{\Theta}^{2}+v^{2}\right)\right]\left\{2 i \omega_{4} v+i\left[h_{\Theta}, \omega_{4}\right]\right\}
\end{aligned}
$$

These integrals are Gaussian and may readily be carried out. The remaining trace (tr) is computed using the eigenstates of $h_{\Theta}$. These manipulations then result in

$$
\begin{aligned}
\mathcal{A}_{E}^{2}= & \frac{N_{C}}{2} T \sum_{\nu} \int_{1 / \Lambda^{2}}^{\infty} \sum_{\nu} \frac{d s}{\sqrt{4 \pi s}} e^{-s\left(\epsilon_{\nu}^{0}\right)^{2}}\left\langle\nu\left|\omega_{4}\right| \nu\right\rangle \\
& -\frac{N_{C}}{4} T \sum_{\nu \mu} \int_{1 / \Lambda^{2}}^{\infty} \frac{d s}{\sqrt{4 \pi s^{3}}} \frac{2+s\left(\epsilon_{\nu}^{0}-\epsilon_{\mu}^{0}\right)^{2}}{\left(\epsilon_{\nu}^{0}\right)^{2}-\left(\epsilon_{\mu}^{0}\right)^{2}}\left(e^{-s\left(\epsilon_{\nu}^{0}\right)^{2}}-e^{-s\left(\epsilon_{\mu}^{0}\right)^{2}}\right)\left\langle\nu\left|\omega_{4}\right| \mu\right\rangle\left\langle\mu\left|\omega_{4}\right| \nu\right\rangle .
\end{aligned}
$$

Making use of closure as well as of the invariance of the second term under $\epsilon_{\mu}^{0} \leftrightarrow \epsilon_{\nu}^{0}$ this expression may be rewritten as

$$
\begin{aligned}
\mathcal{A}_{E}^{2}=\frac{N_{C}}{2} T & \sum_{\mu \nu} \int_{1 / \Lambda^{2}}^{\infty} \frac{d s}{\sqrt{4 \pi s}}\left\langle\nu\left|\omega_{4}\right| \mu\right\rangle\left\langle\mu\left|\omega_{4}\right| \nu\right\rangle \\
& \times\left[\frac{\epsilon_{\mu}^{0} e^{-s\left(\epsilon_{\mu}^{0}\right)^{2}}+\epsilon_{\nu}^{0} e^{-s\left(\epsilon_{\nu}^{0}\right)^{2}}}{\epsilon_{\mu}^{0}+\epsilon_{\nu}^{0}}+\frac{1}{s} \frac{e^{-s\left(\epsilon_{\mu}^{0}\right)^{2}}-e^{-s\left(\epsilon_{\nu}^{0}\right)^{2}}}{\left(\epsilon_{\mu}^{0}\right)^{2}-\left(\epsilon_{\nu}^{0}\right)^{2}}\right]
\end{aligned}
$$

from which $f\left(\epsilon_{\mu}, \epsilon_{\nu} ; \Lambda\right)$ in eqn (5.12) can be extracted after performing an integration by parts in the last term. Next the question arises whether the application of standard perturbation techniques yields the same result. As already discussed in the main part of this paper it does not! In order to see how this comes about we start from the expression of the real part of the action (3.14) and perform the spectral integration

$$
\mathcal{A}_{R}=-\frac{N_{C}}{2} T \sum_{\nu} \int_{1 / \Lambda^{2}}^{\infty} \frac{d s}{\sqrt{4 \pi s^{3}}} \exp \left(-s\left(\epsilon_{\nu}^{R}\right)^{2}\right) .
$$

Using the expansion (5.1) for the energy eigenvalues $\left(\epsilon_{\nu}^{R}=\epsilon_{\nu}^{0}+\epsilon_{\nu}^{2} \ldots\right)$

$$
\tilde{\mathcal{A}}_{E}^{2}=N_{C} T \sum_{\nu} \int_{1 / \Lambda^{2}}^{\infty} \frac{d s}{\sqrt{4 \pi s}} \epsilon_{\nu}^{0} \epsilon_{\nu}^{2} \exp \left(-s\left(\epsilon_{\nu}^{R}\right)^{2}\right)
$$

and substituting the expression (5.2) gives

$$
\begin{aligned}
\tilde{\mathcal{A}}_{E}^{2} & =-\frac{N_{C}}{2} T \sum_{\mu \neq \nu} \frac{\operatorname{sgn}\left(\epsilon_{\nu}^{0}\right)}{\epsilon_{\nu}^{0}-\epsilon_{\mu}^{0}} \operatorname{erfc}\left(\left|\frac{\epsilon_{\nu}^{0}}{\Lambda}\right|\right)\left\langle\nu\left|\omega_{4}\right| \mu\right\rangle\left\langle\mu\left|\omega_{4}\right| \nu\right\rangle \\
& =-\frac{N_{C}}{4} T \sum_{\mu \neq \nu} \frac{\operatorname{sgn}\left(\epsilon_{\nu}^{0}\right) \operatorname{erfc}\left(\left|\frac{\epsilon_{\nu}^{0}}{\Lambda}\right|\right)-\operatorname{sgn}\left(\epsilon_{\mu}^{0}\right) \operatorname{erfc}\left(\left|\frac{\epsilon_{\mu}^{0}}{\Lambda}\right|\right)}{\epsilon_{\nu}^{0}-\epsilon_{\mu}^{0}} .
\end{aligned}
$$

For the energy functional this leads to the expression $\tilde{f}\left(\epsilon_{\mu}, \epsilon_{\nu} ; \Lambda\right)$ given in eqn (5.5). 


\section{References}

[1] For an extensive compilation of articles on soliton models for baryons see, e.g. "Baryons as Skyrme Solitons", G. Holzwarth (Ed.), World Scientific Publ. Comp., Singapore 1993.

[2] S. Brodsky, J. Ellis and M. Karliner, Phys. Lett. B206, 309 (1988);

J. Ellis and M. Karliner, Phys. Lett. B313, 131 (1993);

J. Schechter, A. Subbaraman and H. Weigel, Phys. Rev. D48, 339 (1993).

[3] G. 't Hooft, Nucl. Phys. B72 (1974) 461.

[4] E. Witten, Nucl. Phys. B160 (1979) 57.

[5] T. H. R. Skyrme, Proc. R. Soc. A260 (1661) 127.

[6] G. S. Adkins, C. R. Nappi and E. Witten, Nucl. Phys. B228 (1983) 552.

[7] E. Witten, Nucl. Phys. B223 (1983) 433, 442.

[8] Ö. Kaymakcalan, S. Rajeev and J. Schechter, Phys. Rev. D31 (1985) 1109.

[9] J. Schechter, Phys. Rev. D34 (1986) 868.

[10] M. Bando, T. Kugo and K. Yamawaki, Phys. Rep. 164 (1987) 217.

[11] Ulf-G. Meißner, N. Kaiser and W. Weise, Nucl. Phys. A466 (1987) 685 (1987); Ulf-G. Meißner, Phys. Rep. 161 (1988) 213;

P. Jain, R. Johnson, Ulf-G. Meißner, N. W. Park and J. Schechter, Phys. Rev. D37 (1988) 3252;

Ulf-G. Meißner, N. Kaiser, H. Weigel and J. Schechter, Phys. Rev. D39 (1989) 1956.

[12] J. J. Sakurai, "Currents and Mesons", University of Chicago Press, Chicago 1969.

[13] Y. Nambu and G. Jona-Lasinio, Phys. Rev. 122 (1961) 345; 124 (1961) 246.

[14] For a recent review see e.g. T. Hatsuda and T. Kunihiro, "QCD Phenomenology based on a Chiral Effective Lagrangian". Phys. Rep., to be published.

[15] D. Ebert and H. Reinhardt, Nucl. Phys. B271 (1986) 188.

[16] J. Schwinger, Phys. Rev. 82 (1951) 664.

[17] H. Reinhardt and R. Wünsch, Phys. Lett. B215 (1988) 577; Phys. Lett. B230 (1989) 93 ;

T. Meissner, F. Grümmer and K. Goeke, Phys. Lett. B227 (1989) 296;

R. Alkofer, Phys. Lett. B236 (1990) 310.

[18] R. Alkofer and H. Reinhardt, Phys. Lett. B244 (1991) 461.

[19] R. Alkofer, H. Reinhardt, H. Weigel and U. Zückert, Phys. Rev. Lett. 69 (1992) 1874;

F. Döring, E. Ruiz-Arriola and K. Goeke, Z. Phys. A344 (1992) 159. 
[20] T. Watabe and H. Toki, Prog. Theor. Phys. 87 (1992) 651.

[21] C. Schüren, E. Ruiz-Arriola and K. Goeke, Phys. Lett. B287 (1992) 283.

[22] C. Schüren, F. Döring, E. Ruiz-Arriola and K. Goeke, Nucl. Phys. A565 (1993) 687.

[23] R. Alkofer, H. Reinhardt, H. Weigel and U. Zückert, Phys. Lett. B298 (1993) 132.

[24] U. Zückert, R. Alkofer, H. Reinhardt and H. Weigel, Nucl. Phys. A570 (1994) 445.

[25] S. Kahana and G. Ripka, Nucl. Phys. A429 (1984) 462.

[26] C. Schüren, Contribution at the ETC* workshop on The Quark Structure of Baryons in Trento, October 1993, unpublished.

[27] H. Reinhardt, Nucl. Phys. A503 (1989) 825.

[28] R. Alkofer, H. Reinhardt and H. Weigel, "Baryons as Chiral Solitons in the NambuJona-Lasinio Model", in preparation.

[29] H. Weigel, H. Reinhardt and R. Alkofer, Phys. Lett. B313 (1993) 377.

[30] G. S. Adkins and C. R. Nappi, Phys. Lett. B137 (1984) 251.

[31] N. S. Manton and P. J. Ruback, Phys. Lett. B181 (1986) 137.

[32] M. Wakamatsu and T. Watabe, Phys. Lett. B312 (1993) 184;

A. Blotz, M. Praszalowicz and K. Goeke, Phys. Lett. B317 (1993) 195.

[33] R. Alkofer and H. Weigel, Phys. Lett. B319 (1993) 1.

[34] H. Reinhardt and D. Ebert, Phys. Lett. B173 (1986) 459.

[35] B. Schwesinger, H. Weigel, G. Holzwarth and A. Hayashi, Phys. Rep. 173 (1989) 173.

[36] T. Cohen and M. Banerjee, Phys. Lett. B230 (1989) 129.

[37] H. Weigel, R. Alkofer and H. Reinhardt, Nucl. Phys. B387 (1992) 638;

A. Blotz et al., Nucl. Phys. A555 (1993) 765. 


\section{Figure captions}

\section{Figure 2.1}

Left: The behavior of the valence quarks' eigenenergy in the complex plane $z=\delta e^{i \varphi}$. The starting point $\varphi=0$ is indicated. From there it continues anti-clockwise as $\varphi$ increases. Right: The same for the state with the smallest (positive) real part of the energy eigenvalue in the $G^{\pi}=2^{+}$channel.

\section{Figure 2.2}

The behavior of the valence quarks' eigenfunction in the complex plane in arbitrary units. $\varphi$ labels the phase of the point in the complex plane $z=\delta e^{i \varphi}$. Left: At $r=0$, only the upper component is non-zero. Right: At an arbitrary intermediate point for the case $\delta=1$.

\section{Figure 3.1}

The behavior of the energy $E_{\operatorname{det}}$ (3.24) in the complex plane for the parametrization $z=\delta e^{i \varphi}$. The starting point $\varphi=0$ is indicated. From there the energy continues anti-clockwise.

\section{Figure 5.1}

Left: The total energy as a function of the scaling parameter $\lambda$ defined in eqn (5.17) Right: The parametrical dependence of the energy on the parameter $R_{m}$ as defined in eqn (5.18).

\section{Figure 5.2}

The profile functions which minimize the energy functional (5.14) under variation of the scaling parameter $\lambda$. The quark baryonic density $b \sim q^{\dagger} q(R H S$ of eqn (5.6)) is artificially scaled such that the spatial integrals over $\omega / m$ and $b$ coincide. 
This figure "fig1-1.png" is available in "png" format from: http://arxiv.org/ps/hep-ph/9407304v1 
This figure "fig2-1.png" is available in "png" format from: http://arxiv.org/ps/hep-ph/9407304v1 
This figure "fig1-2.png" is available in "png" format from: http://arxiv.org/ps/hep-ph/9407304v1 
This figure "fig2-2.png" is available in "png" format from: http://arxiv.org/ps/hep-ph/9407304v1 
This figure "fig1-3.png" is available in "png" format from: http://arxiv.org/ps/hep-ph/9407304v1 